\title{
DISTANCE ESTIMATES AND POINTWISE BOUNDED DENSITY
}

\author{
BY
}

\author{
A. M. DAVIE, T. W. GAMELIN AND J. GARNETT( $\left.{ }^{1}\right)$
}

ABSTRACT. Let $U$ be a bounded open subset of the complex plane, and let $H$ be a closed subalgebra of $H^{\infty}(U)$, the bounded analytic functions on $U$. If $E$ is a subset of $\partial U$, let $L_{E}$ be the algebra of all bounded continuous functions on $U$ which extend continuously to $E$, and set $H_{E}=H \cap L_{E}$. This paper relates distance estimates of the form $d(h, H)=d\left(h, H_{E}\right)$, for all $h \in L_{E}$, to pointwise bounded density of $H_{E}$ in $H$. There is also a discussion of the linear space $H+L_{E}$, which turns out often to be a closed algebra.

1. Introduction. In [17] Sarason proved that if $\Delta$ is the open unit disc in the complex plane, then

$$
d(b, A(\Delta))=d\left(b, H^{\infty}(\Delta)\right), \quad \text { all } b \in C(\partial \Delta) .
$$

In [20], Zalcman extended Sarason's theorem to cover algebras of analytic functions on certain infinitely connected planar domains. The object of this paper is to extend the Sarason-Zalcman results to more general open sets $U$, and to consider a wider class of subalgebras of $H^{\infty}(U)$. In order to state our problem and results more precisely, we now fix some notation, which will be used throughout the paper.

By $U$ we will denote a bounded open subset of the complex plane, and by $E$ we will denote an (arbitrary) subset of the topological boundary $\partial U$ of $U$. We let $L(U)$ denote the algebra of bounded continuous function on $U$. The subalgebra of $L(U)$ of bounded analytic functions on $U$ is denoted by $H^{\infty}(U)$. The subalgebra of $L(U)$ of functions which extend continuously to each point of $E$ is denoted by $L_{E}(U)=L_{E}$. Finally we let $H_{E}^{\infty}(U)$ be the algebra of bounded analytic functions on $U$ which extend continuously to each point of $E$ :

$$
H_{E}^{\infty}(U)=H^{\infty}(U) \cap L_{E}
$$

Received by the editors July 9, 1971.

AMS (MOS) subject classifications (1970). Primary 30A82; Secondary 46J15.

Key words and phrases. Bounded analytic functions, pointwise bounded approximation, uniform approximation, distance estimates.

(1) Preparation of this paper was supported in part by NSF Grants GP-19067 and GP.11475. Second author was partially supported by the A. P. Sloan Foundation and NSF grant GP.33693. 
In the particular case $E=\partial U$, we have the algebra $A(U)$ of the continuous functions on $\bar{U}$ which are analytic on $U$ :

$$
A(U)=H_{\partial U}^{\infty}(U)=H^{\infty}(U) \cap C(\bar{U})
$$

Here $C(X)$ denotes the continuous complex-valued functions on $X$. If $B$ is any subspace of $L(U)$, and $b \in L(U)$, then the distance from $b$ to $B$ is given by

$$
d(b, B)=\inf \{\|b-g\|: g \in B\},
$$

the norm being the supremum norm over $U$.

Now let $H$ be any subalgebra of $H^{\infty}(U)$, and set $H_{E}=H \cap L_{E}$. Our problem is the following: How well can we approximate, in the uniform norm, a given function in $L(U)$ by functions in $H_{E}$ ? Since $H_{E} \subseteq H$, we have $d(b, H) \leq d\left(b, H_{E}\right)$ for all $b \in L(U)$. We will be interested in conditions on $U, E$ and $H$ which ensure that

$$
d(b, H)=d\left(b, H_{E}\right), \text { all } b \in L_{E} \cdot
$$

We say that $H_{E}$ is pointwise boundedly dense in $H$ if every function in $H$ can be approximated pointwise on $\dot{U}$ by a bounded sequence in $H_{E}$. It turns out that the validity of the distance estimate $(*)$ is closely related to the pointwise bounded density of $H_{E}$ in $H$, and we will give fairly general conditions under which these notions are equivalent. As a special case of our results (cf. Theorem 5.1), we have the following.

1.1 Theorem. $A(U)$ is pointwise boundedly dense in $H^{\infty}(U)$ if and only if

$$
d(b, A(U))=d\left(b, H^{\infty}(U)\right), \text { all } b \in C(\bar{U}) .
$$

Those sets $U$ for which $A(U)$ is pointwise boundedly dense in $H^{\infty}(U)$ have been characterized by the authors (cf. [2], [13]) in terms of analytic capacity.

Actually, we will prove something much stronger than the forward implication of Theorem 1.1.

1.2 Theorem. Suppose $A(U)$ is pointwise boundedly dense in $H^{\infty}(U)$, and that $E$ is an arbitrary subset of $\partial U$. Then

$$
d\left(b, H_{E}^{\infty}(U)\right)=d\left(b, H^{\infty}(U)\right), \text { all } b \in L_{E} .
$$

Our original motivation for studying problems of this sort was to approximate uniformly functions in $H_{E}^{\infty}(U)$ by functions in $H^{\infty}(U)$ which extend analytically across $E$ (cf. [14]). An example of the approximation theorem we have in mind is the following

1.3 Corollary. Suppose that $A(U)$ is pointwise boundedly dense in $H^{\infty}(U)$, and that $E$ is an arbitrary subset of $\partial U$. Then every $f \in H_{E}^{\infty}(U)$ can be 
approximated uniformly on $U$ by functions in $H^{\infty}(U)$ which extend continuously to an open set containing $E$.

Proof. Let $\epsilon>0$. Since $f \in L_{E}$, there is an open subset $V$ of $\partial U$ and $b \in L_{V}$ such that $\|b-f\|<\epsilon / 2$. Then $d\left(b, H^{\infty}(U)\right)<\epsilon / 2$. By Theorem 1.2 , there is $g \in H_{V}^{\infty}(U)$ such that $\|g-b\|<\epsilon / 2$. This $g$ approximates $f$ within $\epsilon$.

The paper begins with the derivation of pointwise bounded density from the distance estimate in $\$ 2$. The converse assertion is somewhat more difficult and can only be proved under certain hypotheses, which are discussed in $\$ 3$. The proof of the converse is in $\$ \$ 4$ through 6. A similar distance estimate is considered in $\$ 7$. "Geometric" conditions which yield the distance estimates are given in $\$ 8$.

The results described so far do not directly generalize the results of Sarason and Zalcman because we consider algebras on $\bar{U}$ rather than $\partial U$. In $\$ 9$ we prove analogous results for the algebra $H^{\infty}(\mu)$, the weak-star closure of $A(U)$ in $L^{\infty}(\mu)$, where $\mu$ denotes harmonic measure on $\partial U$ for $U$. This includes the desired generalization. We also consider $H^{\infty}(\sigma)$ where $\sigma$ denotes area measure on $U$.

In $\$ 10$ we turn to estimating the distance of $f \in H$ to $H_{E}$ in terms of its distance to $L_{E}$. In $\$ 11$ we consider the subspace $H+L_{E}$ of $L(U)$, which turns out to be a closed subalgebra of $L(U)$ under suitable hypotheses. In $\$ 12$ we specialize to the case of the unit disc, discussing the subspaces $H^{\infty}(d \theta)+L_{E}^{\infty}(d \theta)$ of $L^{\infty}(d \theta)$. We prove these linear spaces are always closed subalgebras of $L^{\infty}(d \theta)$, which are generated by $H^{\infty}(d \theta)$ and the complex conjugates of Blaschke products which are analytic on $E$. Some open problems are mentioned in $\$ 13$.

Some more notation: the open disc with center at $z$ and radius $r$ will be denoted by $\Delta(z ; r)$. All norms will be supremum norms, unless otherwise indicated. The supremum norm over a set $S$ is denoted by $\|\cdot\|_{S}$ :

$$
\|f\|_{S}=\sup \{|f(x)|: x \in S\} .
$$

The term "measure" will mean "complex regular Borel measure." If $A$ is a set of continuous functions on a compact set $X$ and $\mu$ is a measure on $X$, we write $\mu \perp A$ if $\int f d \mu=0$ for all $f \in A$. We denote by $A^{\perp}$ the set of measures $\mu$ on $X$ for which $\mu \perp A$.

2. Distance estimate implies pointwise bounded density. Let $\mathbb{M}$ be the Stone-Čech compactification of $U$ and let $\pi_{E}$ be that of $U \cup E$. Then $L(U) \cong C(\pi)$ and $L_{E} \cong C\left(\pi_{E}\right)$. The inclusion $L_{E} \rightarrow L(U)$ induces a map $\pi: \pi \rightarrow \pi_{E}$ which is the identity on $U$.

The coordinate function $Z$ projects $M$ onto the closure $\bar{U}$ of $U$. If $\lambda \in E$, 
then the projection $\pi$ collapses the fiber $Z^{-1}(\lambda)$ to a point. If $\lambda$ is not in the closure of $E$, then the projection $\pi$ is a homeomorphism on the fiber $Z^{-1}(\lambda)$. In particular, if $E$ is closed, we can describe $\pi$ completely: it collapses each fiber over $E$ to a point, and it maps $M \backslash Z^{-1}(E)$ homeomorphically onto $M_{E} \backslash E$.

We can regard $\pi$ as a map from measures on $\mathbb{M}$ to measures on $\mathbb{M}_{E}$, by setting $(\pi \mu)(S)=\mu\left(\pi^{-1}(S)\right)$, whenever $S$ is a Borel subset of $\mathbb{M}_{E}$. Equivalently, $\int b d \mu=\int b d(\pi \mu), b \in L_{E}(\mu)$. Recall that $H_{E}=H \cap L_{E}$, whenever $H$ is a closed subalgebra of $H^{\infty}(U)$. The preceding identity shows that $\pi\left(H^{\perp}\right) \subseteq H_{E}^{\perp}$.

2.1 Lemma. Let $H$ be a closed subalgebra of $H^{\infty}(U)$ and let $E$ be a subset of $\partial U$. Then the following are equivalent:

(i) $d\left(b, H_{E}\right)=d(b, H)$ for all $b \in L_{E}$;

(ii) for each measure $\nu$ on $\mathbb{M}_{E}$ orthogonal to $H_{E}$ there exists a measure $\mu$ on $\pi$ orthogonal to $H$ such that $\pi(\mu)=\nu$ and $\|\mu\|=\|\nu\|$.

Proof. Assume (i) is true. Let $\nu \in H_{E}^{\perp}$ satisfy $\|\nu\|=1$. Define a linear functional $\Lambda$ on the linear subspace $H+L_{E} \subseteq L(U)$ by

$$
\Lambda(f+b)=\int b d \nu, \quad f \in H, b \in L_{E} .
$$

This functional is well defined because $\nu$ annihilates $H_{E}=H \cap L_{E}$. If $\|f+b\| \leq 1$ then $d(b, H) \leq 1$, so that $d\left(b, H_{E}\right) \leq 1$ and $\left|\int b d \nu\right| \leq 1$. Thus $\Lambda$ has norm 1. Let $\mu$ be a Hahn-Banach extension of $\Lambda$ to $L(U)$. Then $\mu \in H^{\perp}$, $\pi(\mu)=\nu$ and $\|\mu\|=1$.

Conversely, suppose (ii) is true. Let $b \in L_{E}$. By the Hahn-Banach theorem, there is a measure $\nu \in H_{E}^{\perp}$ such that $d\left(b, H_{E}\right)=\int b d \nu$ and $\|\nu\|=1$. Let $\mu$ be the lift of $\nu$ given by (ii). Then $d\left(b, H_{E}\right)=\int b d \mu \leq d(b, H)$. That completes the proof.

In the same way one can show that if $c \geq 1$, then $d\left(b, H_{E}\right) \leq c d(b, H)$ for all $b \in L_{E}$ if and only if each $\nu \in H_{E}^{\perp}$ has a lift $\mu \in H^{\perp}$ with $\|\mu\| \leq c\|\nu\|$.

2.2 Theorem. Let $H$ be a closed subalgebra of $H^{\infty}(U)$ and let $E$ be a sub. set of $\partial U$. Suppose that $d\left(b, H_{E}\right)=d(b, H)$, for all $b \in L_{E}$. Then for any $f \in H$ we can find a sequence $\left\{f_{n}\right\}$ in $H_{E}$ such that $\left\|f_{n}\right\| \leq\|f\|$ and $f_{n}$ converges to $f$ uniformly on each subset of $U$ with positive distance from $E$.

Proof. Let $f \in \operatorname{Ball}(H)$. We have to show that $f$ is in the closure of Ball $\left(H_{E}\right)$ in the topology of uniform convergence on compact subsets of $M \backslash Z^{-1}(\bar{E})$. By the Hahn-Banach theorem it suffices to show that if $K$ is a compact subset of $M \backslash Z^{-1}(\bar{E})$ and $\sigma$ is a measure on $K$ such that 


$$
\left|\int g d \sigma\right| \leq 1 \text { for all } g \in \operatorname{Ball}\left(H_{E}\right)
$$

then $\left|\int f d \sigma\right| \leq 1$.

So let $\sigma$ be as above, and let $\eta$ be a norm-preserving extension to $L_{E}$ of the functional $g \rightarrow \int g d \sigma$ on $H_{E}$. Then $\|\eta\| \leq 1$ and the measure $\nu=\eta-\sigma$ is orthogonal to $H_{E^{*}}$

Using Lemma 2.1, take $\mu \in H^{\perp}$ such that $\|\mu\|=\|\nu\|$ and $\pi(\mu)=\nu$. Since $\pi$ is norm-decreasing we have $|\nu|(J) \leq|\mu|\left(\pi^{-1}(J)\right)$ for any Borel set $J \subseteq \mathbb{M}_{E}$. Since $\|\nu\|=\|\mu\|$ we must have $|\nu|(J)=|\mu|\left(\pi^{-1}(J)\right)$. Applying this to $\left.J=\pi(\pi) \backslash K\right)$ we obtain $|\mu+\sigma|(\pi \backslash K)=|\mu|(\mathcal{N} \backslash K)=|\nu|\left(\pi_{E} \backslash K\right)=|\eta|\left(\pi_{E} \backslash K\right)$. Since $\pi$ is one-to-one on $K$ we have $|\mu+\sigma|(K)=|\pi(\mu+\sigma)|(K)=|\eta|(K)$. These inequalities. show that $\|\mu+\sigma\| \leq\|\eta\|=1$ so that

$$
\left|\int f d \sigma\right|=\left|\int f d(\mu+\sigma)\right| \leq 1
$$

as required.

3. Stable algebras. Now we will begin seeking conditions under which pointwise bounded density implies the distance estimate. In this section, we define a class of subalgebras of $H^{\infty}(U)$ to which our techniques apply.

A subalgebra $H$ of $H^{\infty}(U)$ is stable if the following conditions are met:

(i) The uniform closure of $H$ contains $A(U)$.

(ii) If $f \in H$ and $z_{0} \in U$, then $\left[f-f\left(z_{0}\right)\right] /\left(z-z_{0}\right) \in H$.

Examples of stable subalgebras of $H^{\infty}(U)$ include $A(U)$, and $H^{\infty}(U)$ itself. In fact, if $H$ is stable, then so is $H_{E}$, so that all the algebras $H_{E}^{\infty}(U)$ are stable. Another important class of stable algebras are the algebras $H_{E}^{\infty}(\sigma)$, where $H^{\infty}(\sigma)$ is the weak-star closure of $A(U)$ in $L^{\infty}(\sigma)$, and $\sigma$ is the area measure on $U$.

For the remainder of this section, we fix a closed stable subalgebra $H$ of $H^{\infty}(U)$. The maximal ideal space of $H$ is denoted by $\Re(H)$. By identifying each $\lambda \in U$ with the homomorphism "evaluation at $\lambda$ ", we can regard $U$ as a subset of $\pi(H)$. When convenient we will regard the functions in $H$ as continuous functions on $\Re(H)$. With this convention, the coordinate function $Z$ projects $M(H)$ onto a compact subset of the complex plane. Since $1 /\left(z-z_{0}\right) \in H$ if and only if $z_{0} \notin \bar{U}, Z$ projects $\mathbb{M}(H)$ onto $\bar{U}$. For each $\lambda \in \bar{U}$, the set $Z^{-1}(\lambda) \subseteq$ $\pi(H)$ is called the "fiber" over $\lambda$, and denoted by $\Re_{\lambda}(H)$. Using (ii), it is easy to see that $\mathbb{M}_{\lambda}(H)$ consists of only the point $\{\lambda\}$ whenever $\lambda \in U$.

By Arens' theorem, the maximal ideal space of $A(U)$ coincides with $\bar{U}$. The inclusion $A(U) \hookrightarrow H$ induces a projection $\Re(H) \rightarrow \pi(A(U))=\bar{U}$, which coincides with the coordinate map $Z$. In particular, every function in $A(U)$ is constant on each fiber $M_{\lambda}(H)$.

Let $g$ be a smooth function with compact support. If $f$ is a bounded Borel function, we define 


$$
\begin{aligned}
& \left(R_{g} f\right)(\zeta)=\frac{1}{\pi} \iint \frac{f(z)}{z-\zeta} \frac{\partial g}{\partial \bar{z}} d x d y, \\
& \left(T_{g} f\right)(\zeta)=\frac{1}{\pi} \iint \frac{f(z)-f(\zeta)}{z-\zeta} \frac{\partial g}{\partial \bar{z}} d x d y=g(\zeta) f(\zeta)+\left(R_{g} f\right)(\zeta)
\end{aligned}
$$

Since $R_{g} f$ is the convolution of a bounded function with the locally integrable function $1 / z, R_{g} f$ is continuous. Consequently $T_{g} f$ extends continuously to any point at which $f$ is continuous. In the sense of distributions, we have $(\partial / \partial \bar{z}) T_{\boldsymbol{g}} f=g \partial f / \partial \bar{z}$. It follows that $T_{\boldsymbol{g}} f$ is analytic wherever $f$ is, $T_{\boldsymbol{g}} f$ is analytic off the closed support of $g$, and $f-T_{\boldsymbol{g}} f=R_{\boldsymbol{g}} f$ is analytic on the interior of the level set $g^{-1}(1)$ of $g$.

If $f \in H^{\infty}(U)$, we extend $f$ to be zero off $U$, and define $T_{g} f$ as above. Then $T_{g} f \in H^{\infty}(U)$. The key property of closed stable algebras is their invariance under the operators $T_{g^{*}}$

3.1 Lemma. Let $H$ be a closed stable subalgebra of $H^{\infty}(U)$, let $f \in H$ and let $g$ be a smooth function with compact support. Then $T_{g} f \in H$. The "Gelfand" extension of $T_{g} f$ from $U$ to $\mathbb{M}(H)$ is obtained by declaring $g=g(\lambda)$ and $R_{g} f=R_{g} f(\lambda)$ on each fiber $\mathbb{M}_{\lambda}(H)$ :

$$
\left(T_{g} f\right)(\phi)=g(\lambda) f(\phi)+\left(R_{g} f\right)(\lambda), \quad \phi \in \mathbb{M}_{\lambda}(H)
$$

Proof. Define

$$
F(\zeta)=\frac{1}{\pi} \iint_{U} \frac{f(z)-f(\zeta)}{z-\zeta} \frac{\partial g}{\partial \bar{z}} d x d y, \quad \zeta \in U
$$

For each $z \in U,[f(z)-f(\zeta)] /(z-\zeta)$ belongs to $H$. The integrals $\iint|z-\zeta|^{-1}|\partial g / \partial z| d x d y$ converge uniformly in $\zeta \in U$. Regarding $F$ as the integral of an $H$-valued function, we see that the integral $F$ belongs to $H$. Now

$$
G(\zeta)=\frac{1}{\pi} \iint_{G \backslash U} \frac{1}{z-\zeta} \frac{\partial g}{\partial \bar{z}} d x d y
$$

defines a function $G \in A(U)$ satisfying $T_{\boldsymbol{g}} f=F-f G$. Consequently $T_{\boldsymbol{g}} f \in H$. If $\phi \in \mathbb{M}_{\lambda}(H)$, then

$$
\begin{aligned}
\phi\left(T_{g} f\right) & =\phi(F)-\phi(f) \phi(G) \\
& =\frac{1}{\pi} \iint_{U} \phi\left(\frac{f(z)-f(\zeta)}{z-\zeta}\right) \frac{\partial g}{\partial \bar{z}} d x d y-\phi(f) G(\lambda) \\
& =\frac{1}{\pi} \iint_{U} \frac{f(z)-\phi(f)}{z-\lambda} \frac{\partial g}{\partial \bar{z}} d x d y-\frac{\phi(f)}{\pi} \iint \frac{1}{z-\lambda} \frac{\partial g}{\partial \bar{z}} d x d y \\
& =\left(R_{g} f\right)(\lambda)+\phi(f) g(\lambda) .
\end{aligned}
$$


That completes the proof.

3.2 Lemma. Let $\lambda \in \partial U$, and let $g$ be a smooth function supported on the disc $\Delta(\lambda ; \delta)$ satisfying $0 \leq g \leq 1$ and $|\partial g / \partial \bar{z}| \leq 4 / \delta$. Then for $f \in H^{\infty}(U)$,

$$
\left\|T_{g} f\right\| \leq 8 \sup \{|f(z)-f(\zeta)|: z, \zeta \in \Delta(\lambda ; \delta)\} .
$$

Proof. The norm of $T_{g} f$ is attained over $\Delta(\lambda ; \delta)$, because $T_{g} f$ is analytic off that disc. With this in mind the estimate follows easily from the inequality $\iint_{\Delta(\lambda ; \delta)}|z-\zeta|^{-1} d x d y \leq 2 \pi \delta$.

The next result is a weak form of the cluster value theorem valid for $H^{\infty}(U)[11]$.

3.3 Theorem. Let $H$ be a closed stable subalgebra of $H^{\infty}(U)$ and let $\lambda \in \partial U$. If $f \in H$, then

$$
\|f\|_{\pi_{\lambda}(H)}=\limsup _{U \ni \zeta \rightarrow \lambda}|f(\zeta)|
$$

Proof. Clearly by compactness of $\mathbb{M}(H)$,

$$
\limsup _{U \ni \zeta \rightarrow \lambda}|f(\zeta)| \leq\|f\|_{\pi_{\lambda}(H)}
$$

Suppose there is a disc $\Delta(\lambda ; \delta)$ such that $|f| \leq 1$ on $U \cap \Delta(\lambda ; \delta)$. We must show $\|f\|_{\pi_{\lambda}(H)} \leq 1$. Let $g$ be as in 3.2. Then $\left\|T_{g} f\right\|_{\pi_{\lambda}(H)} \leq 16$, and the inequality in the proof of 3.2 shows that $\left|R_{g} f(\lambda)\right| \leq 8$. By $3.1,|f| \leq 24$ on $\mathbb{M}_{\lambda}(H)$. The same argument shows $\left|f^{n}\right| \leq 24$ on $\mathbb{M}_{\lambda}(H)$ for every positive integer $n$. Taking $n$th roots and letting $n$ tend to $\infty$, we obtain $|f| \leq 1$ on $\mathbb{M}_{\lambda}(H)$.

3.4 Corollary. Let $H$ be a closed stable subalgebra of $H^{\infty}(U)$. If $f \in H$ extends continuously to $\lambda$ (from $U)$, then $f$ is constant on $\Pi_{\lambda}(H)$.

3.5 Theorem. Let $H$ be a closed stable subalgebra of $H^{\infty}(U)$, and let $\lambda \in \partial U$. If $f$ belongs to the restriction algebra $\left.H\right|_{\pi_{\lambda}(H)}$, then there is $F \in H$ such that $\left.F\right|_{\pi_{\lambda}(H)}=f$ and $\|F\|_{U} \leq 33\|f\|_{\pi_{\lambda}(H)^{*}}$.

Proof. Suppose $\|f\|<1$. Choose $F_{0} \in H$ such that $\left.F_{0}\right|_{M_{\lambda}(H)}=f$. Then $\left|F_{0}\right|<1$ on $\Delta(\lambda ; \delta) \cap U$ for some $\delta>0$. Choose $g$ as in Lemma 3.2, with $g=1$ in a neighborhood of $\lambda$, and set $F=T_{g} F_{0}-\left(R_{g} F_{0}\right)(\lambda) \in H$. Then $F-F_{0}$ tends to zero at $\lambda$. By Corollary 3.4,F coincides with $f$ on $M_{\lambda}(H)$. Using 3.2 to estimate $T_{g} F_{0}$, and 3.3 to estimate $\left(R_{g} F_{0}\right)(\lambda)$, we obtain $\|F\|<33$.

3.6 Corollary. If $H$ is a closed stable subalgebra of $H^{\infty}(U)$ and $\lambda \in \partial U$, then the restriction algebra $\left.H\right|_{\pi_{\lambda}(H)}$ is uniformly closed.

4. A separation lemma. In this section we prove the version of the separation theorem for convex sets which we need later. 
4.1 Lemma. Let $\mathfrak{Y}$ be a closed subspace of the real Banach space $\mathfrak{X}$ and let $\phi$ be a continuous linear functional on $\vartheta$. Let $\Omega$ be a bounded convex open subset of $\mathfrak{X}$, let $\epsilon>0$, and let $\Omega_{\epsilon}$ be the $\epsilon$-neighborbood of $\Omega$ in $\mathfrak{X}$. Suppose $a \in \mathbf{R}$ satisfies $\phi(y)<a$ for all $y \in \mathbb{V} \cap \Omega_{\epsilon}$. Then there exists a continuous

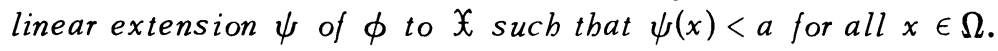

Proof. Replacing $X$ and $\mathscr{V}$ respectively by $X / \phi^{-1}(0)$ and $\mathscr{V} / \phi^{-1}(0)$ we may assume that $\mathscr{V}$ is one-dimensional. First suppose that $\vartheta \cap \bar{\Omega}_{\epsilon / 2} \neq \varnothing$. Let $x_{0}$ be the unique point of $\mathscr{V}$ with $\phi\left(x_{0}\right)=a$. Since $x_{0} \notin \bar{\Omega}_{\epsilon / 2}$, we can find a continuous linear functional $\theta$ on $X$ with $\theta\left(x_{0}\right)>\sup \left\{\theta(x): x \in \bar{\Omega}_{\epsilon / 2}\right\}$. Then $\left.\theta\right|_{\mathfrak{D}}=c \phi$ for some $c \in \mathbf{R}_{0}$. Since $\bar{\Omega}_{\epsilon / 2} \cap \mathcal{V} \neq \varnothing$, we must have $c>0$. Then $\psi=\theta / c$ is the required extension.

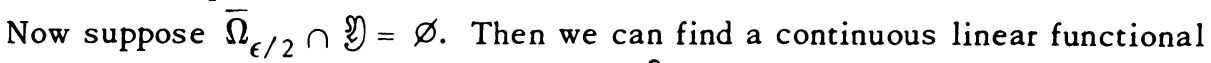
$\rho$ on $\mathfrak{X}$ with $\left.\rho\right|_{\mathfrak{D}}=0$ and $\rho<-1$ on $\Omega$. Let $\tilde{\phi}$ be any continuous extension of

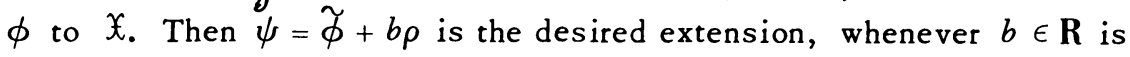
sufficiently large.

5. The distance estimate for closed sets. In this section we establish the following theorem, which provides a converse for 2.2 in the case that $E$ is closed.

5.1 Theorem. Let $H$ be a closed stable subalgebra of $H^{\infty}(U)$, and let $E$ be a closed subset of $\partial U$. The following are equivalent:

(i) $d\left(b, H_{E}\right)=d(b, H)$, for all $b \in L_{E}$.

(ii) $H_{E}$ is pointwise boundedly dense in $H$.

(iii) If $f \in H$, then there is a sequence $\left\{f_{n}\right\}$ in $H_{E}$ such that $\left\|f_{n}\right\| \leq\|f\|$, and $f_{n}$ converges uniformly to $f$ on each subset of $U$ at a positive distance from $E$.

Proof. Theorem 2.2 shows that (i) implies (iii), while (iii) evidently implies (ii). So we must show that (ii) implies (i). This will follow immediately from Lemma 5.3. First we prove the following lemma, which will be used again in the next section.

5.2 Lemma. Let $H$ be a closed stable subalgebra of $H^{\infty}(U)$, let $E$ and $T$ be disjoint compact subsets of $\partial U$, and let $f \in H_{T}$. Suppose there is a bounded sequence $\left\{f_{n}\right\}$ in $H_{E}$ converging pointwise to $f$ on $U$. Then we can find a bounded sequence $\left\{F_{n}\right\}$ in $H_{E \cup T}$ converging uniformly to $f$ on each subset of $U$ at positive distance from $E$.

Proof. Let $M=\sup \left\|f_{n}\right\|$. Let $S \subseteq U$ be at a positive distance from $E$, and let $\epsilon>0$. It suffices to find $F \in H_{E \cup T}$ satisfying $\|F\|<2 M+\epsilon$ and $|F-f|<\epsilon$ on $S$.

Let $g$ be a continuously differentiable function on $\mathbf{C}$, with $0 \leq g \leq 1$, such 
that $g=0$ on a neighborhood of $E$ and $g=1$ on a neighborhood of $\bar{S} \cup T$. For each $n$ let $\psi_{n}=T_{g}\left(f-f_{n}\right)$, so that

$$
\psi_{n}(\zeta)=g(\zeta)\left(f(\zeta)-f_{n}(\zeta)\right)+\frac{1}{\pi} \iint_{U} \frac{f(z)-f_{n}(z)}{z-\zeta} \frac{\partial g}{\partial \bar{z}} d x d y, \quad \zeta \in U .
$$

Then $\psi_{n} \in H_{E}$. By Hölder's inequality,

$$
\left\|\psi_{n}-g\left(f-f_{n}\right)\right\|_{\infty} \leq \frac{1}{\pi}\left\|\frac{\partial g}{\partial \bar{z}}\right\|_{\infty}\left\|f-f_{n}\right\|_{3} \sup _{\zeta \in U}\left\|\frac{1}{z-\zeta}\right\|_{3 / 2},
$$

where the $L^{p}$-norms are with respect to the area measure on $U$. Now $f_{n}$ converges to $f$ in $L^{3}$, and the last factor is bounded by a constant depending only on the diameter of $U$, so we can choose $N$ so that $\left\|\psi_{N}-g\left(f-f_{N}\right)\right\|<\epsilon$. Then $F=f_{N}+$ $\psi_{N}$ has the desired properties. That completes the proof of the lemma.

5.3 Lemma. Let $E$ be a compact subset of $\partial U$. Let $B$ and $H$ be closed stable subalgebras of $H^{\infty}(U)$ such that $B \subseteq H_{E}^{\infty}(U)$. Suppose that for eacb $f \in H$ there exists a sequence $\left\{f_{n}\right\}$ in $B$ with sup $\left\|f_{n}\right\|<\infty$ and $f_{n} \rightarrow f$ uniformly on each subset of $U$ at a positive distance from $E$. Let $b_{1}, \cdots, b_{k} \in L_{E}$, let $X_{1}, \cdots, X_{k}$ be compact subsets of $\bar{U}$, and let $\eta_{1}, \cdots, \eta_{k}>0$. Let $N$ be the set of bounded continuous functions $f$ on $U$ such that $\left\|f-b_{i}\right\|_{V_{i} \cap U}<\eta_{i}$ for some neighborbood $V_{i}$ of $X_{i}, 1 \leq i \leq k$. Let $f \in H \cap N$. Then we can find a sequence $\left\{F_{n}\right\}$ in $B \cap N$ such that $F_{n} \rightarrow f$ uniformly on every subset of $U$ at positive distance from $E$.

Before proceeding to the proof of Lemma 5.3, note that the implication (ii) $\Rightarrow$ (i) of Theorem 5.1 follows immediately from Lemmas 5.2 and 5.3. For this, we use only the special case of Lemma 5.3 in which $B=H_{E}, k=1, b_{1}=b$, $X_{1}=\bar{U}$, and $\eta_{1}=d(b, H)+\epsilon$. However, we will need the more technical statement in the next section in order to extend the result to nonclosed sets.

Proof of Lemma 5.3. Recall that $\mathbb{M}_{E}$ is the Stone-Čech compactification of $U \cup E$ so that $L_{E} \cong C\left(\pi_{E}\right)$. Uniform convergence on each subset of $U$ at a positive distance from $E$ is the same as uniform convergence on compact subsets of $\pi_{E} \backslash E$. Hence we must show that $F$ lies in the closure of $B \cap N$ in the topology of uniform convergence on compact subsets of $\mathbb{M}_{E} \backslash E$. Let $\mu$ be a measure with compact support in $\Re_{E} \backslash E$ and let

$$
a>\sup \left\{\operatorname{Re} \int f d \mu: f \in B \cap N\right\} \text {. }
$$

Since $N$ is convex it suffices to show that $\operatorname{Re} \int F d \mu \leq a$.

We can find $\eta_{i}^{\prime}<\eta_{i}$ for each $i$ such that $F \in N^{\prime}$, where $N^{\prime}$ is defined like $N$ with $\eta_{i}^{\prime}$ in place of $\eta_{i}$. Define a continuous real-linear functional $\phi$ on $B$ by $\phi(f)=\operatorname{Re} \int f d \mu, f \in B$. Using Lemma 4.1 with $\Omega=N^{\prime}$, we extend $\phi$ to $L_{E}$ and 
obtain a measure $\nu$ on $\mathbb{M}_{E}$ such that $\operatorname{Re} \int g d \nu \leq a$ for all $g \in N^{\prime} \cap L_{E}$, while $\operatorname{Re} \int f d \nu=\operatorname{Re} \int f d \mu$ for all $f \in B$. Then $\nu-\mu$ is orthogonal to $B$.

Let $\mathcal{E}$ be the algebra obtained from the algebra of bounded Borel functions on $\pi_{E}$ which are continuous on $\Re_{E} \backslash E$, by identifying any two functions which coincide on $\mathbb{M}_{E} \backslash E$ and coincide $|\nu|$-almost everywhere on $E$. Then $\mathcal{E}$ is essentially the direct sum of $L^{\infty}\left(\left|\nu_{E}\right|\right)$ and the algebra of bounded continuous functions on $\mathbb{M}_{E} \backslash E$. Note that $B \subseteq L_{E} \subseteq \mathcal{E}$. We introduce a topology $\tau$ on $\mathcal{E}$ by declaring that a net $\left\{f_{a}\right\}$ in $\mathcal{E}$ converges to $f \in \mathcal{E}$ in the $\tau$-topology if and only if $f_{a}$ converges uniformly to $f$ on every compact subset of $\prod_{E} \backslash E$ and $f_{a}$ converges weakstar to $f$ in $L^{\infty}(|\nu|)$.

Let $\mathcal{F}$ be the $\tau$-closure of $B$. Then $\mathcal{F}$ is a $r$-closed subalgebra of $\mathcal{E}$, and the functions in $\mathcal{F}$ are all bounded and analytic on $U$. Since $\mu-\nu \perp B$, and $\tau$-convergence implies weak-star convergence in $L^{\infty}(|\mu-\nu|)$, we also have $\mu-\nu \perp \mathcal{F}$.

Let $\rho$ be the restriction map $\rho(b)=\left.b\right|_{U}, b \in \mathcal{E}$. Then $\rho(\mathcal{E})$ is the algebra of all bounded continuous functions on $U$, and $\rho(\mathfrak{F})$ is a closed subalgebra of $H^{\infty}(U)$.

The remainder of the proof will be decomposed into four lemmas.

5.4 Lemma. If $f \in \mathfrak{F}$ vanishes on $U$ then $f=0$.

Proof. Since $f$ vanishes on $\mathbb{M}_{E} \backslash E$ it will suffice to show $f=0$ almost everywhere $(d|\nu|)$. Let the net $\left\{f_{\alpha}\right\}$ in $B$ converge to $f$ in the $\tau$-topology, and let $z_{0} \in U$. Since $B$ is stable, the function $\left[f_{a}(z)-f_{a}\left(z_{0}\right)\right] /\left(z-z_{0}\right)$ is in $B$. Noting that $f_{a}\left(z_{0}\right) \rightarrow f\left(z_{0}\right)=0$, it is easily verified that $\left[f_{a}(z)-f_{a}\left(z_{0}\right)\right] /\left(z-z_{0}\right)$ converges to $f(z) /\left(z-z_{0}\right)$ in the $\tau$-topology. Hence

$$
\int \frac{f(z)}{z-z_{0}} d(\nu-\mu)=0, \quad z_{0} \in U .
$$

Since $f=0$ almost everywhere $(d|\mu|)$, we have $f d \nu \perp B \supseteq A(U)$ and $f d \nu \perp$ $1 /\left(z-z_{0}\right)$ for all $z_{0} \in U$. Now the linear span of $A(U)$ and the functions $1 /\left(z-z_{0}\right), z_{0} \in U$, is dense in $C(\partial U)$ (cf. [2]). Since $f d \nu$ is carried by $E \subseteq \partial U$, we must have $f d \nu=0$, so $f=0$ almost everywhere $(d|\nu|)$, as required.

5.5 Lemma. The restriction map $\rho$ maps $\rho^{-1}(H) \cap \mathcal{F}$ isometrically onto $H$.

Proof. First, $\rho(\mathcal{F}) \supseteq H$. To see this, let $f \in H$ and let $\left\{f_{n}\right\}$ be a bounded sequence in $B$ converging to $f$ uniformly on subsets of $U$ at a positive distance from $E$. If $\left\{f_{a}\right\}$ is a subnet of $\left\{f_{n}\right\}$ which converges in the weak-star topology of $L^{\infty}(|\nu|)$, then $f_{a}$ converges in the $\tau$-topology to a function in $\mathcal{F}$ whose restriction to $U$ coincides with $f$.

Now $\rho$ maps $\rho^{-1}(H) \cap \mathcal{F}$ one-to-one onto $H$. By the closed graph theorem, 
$\rho$ is bicontinuous. Moreover, $\rho$ is multiplicative, and hence is an isomorphism of the uniform algebras $\rho^{-1}(H) \cap \mathcal{F}$ and $H$. Since any isomorphism of uniform algebras is an isometry, the lemma is established.

5.6 Lemma. Suppose $b \in \mathcal{F}$ satisfies $\rho(b)=F$. Then

$$
\left\|b-b_{i}\right\|_{L}^{\infty}\left(|\nu| V_{i} \cap E^{\prime}<\eta_{i}^{\prime}, \quad 1 \leq i \leq k .\right.
$$

Proof. Let $\phi$ be a complex-valued homomorphism of $L^{\infty}(|\nu|)$ lying in the fiber over $\lambda \in E$, i.e., satisfying $\phi(Z)=\lambda$. The restriction of $\phi$ to $\mathfrak{F} \cap \rho^{-1}(H)$ determines a homomorphism $\tilde{\phi}$ of $H$ satisfying $\tilde{\phi}(Z)=\lambda$ and $\phi(f)=\tilde{\phi}(\rho(f))$, $f \in \mathcal{F} \cap \rho^{-1}(H)$. If $\lambda \in V_{i}$ then the cluster values of $F-b_{i}(\lambda)$ at $\lambda$ are bounded by $\left\|F-b_{i}\right\|_{V_{i}}$, so that by Theorem 3.3 we have $\left|\tilde{\phi}\left(F-b_{i}(\lambda)\right)\right| \leq\left\|F-b_{i}\right\|_{V_{i}}$, whence $\left|\phi(b)-b_{i}(\lambda)\right| \leq\left\|F-b_{i}\right\|_{V_{i}}$. Since $\phi(g)=g(\lambda)$ for all $g \in L_{E}$, we have $\left|\phi\left(b-b_{i}\right)\right| \leq\left\|F-b_{i}\right\|_{V_{i}}$. Since this is true for all $\phi$ in fibers over $E \cap V_{i}$, the norm of $b-b_{i}$ in $L^{\infty}\left(|\nu|_{V_{i} \cap E}\right)$ is bounded by $\left\|F-b_{i}\right\|_{V_{i}}<\eta_{i}^{\prime}$, as required.

5.7 Lemma. Let $b \in \mathcal{F}$ satisfy $\rho(b)=F$. Then $b$ is in the $\tau$-closure of $N^{\prime} \cap L_{E^{*}}$

Proof. This is a straightforward consequence of the estimates obtained in Lemma 5.6. It can be proved as follows. Choose $\delta>0$ so that $2 \delta<\eta_{i}^{\prime}-$ $\left\|F-b_{i}\right\|_{V_{i} \cap U}, 1 \leq i \leq k$. Let $W_{1}, \cdots, W_{r}$ be an open cover of $\mathbb{M}_{E}$ such that the oscillation of each $b_{i}$ on $W_{j}$ is less than $\delta$. We can assume that either $\overline{\pi\left(W_{j}\right) \subseteq}$ $V_{i}$ or $\overline{\pi\left(W_{j}\right)} \cap X_{i}=\varnothing$ for each pair $i, j$ (here $\pi$ is the projection of $\mathbb{M}_{E}$ onto $\bar{U}$ ). Fix a point $x_{j} \in W_{j}, 1 \leq j \leq r$, and let $T_{i j}$ be the open disc $\left\{\left|\zeta-b_{i}\left(x_{j}\right)\right|<\eta_{i}^{\prime}\right\} \subseteq \mathbf{C}$. For each $j$ set

$$
T_{j}=\bigcap\left\{T_{i j}: i \text { such that } \overline{\pi\left(W_{j}\right)} \subset V_{i}\right\} .
$$

Then $T_{j}$ is a convex open set in $\mathrm{C}$, and $b(x) \in T_{j}$ for all $x \in W_{j} \backslash E$ and $|\nu|-$ almost all $x \in W_{j} \cap E$. Consequently there is a bounded sequence $\left\{\psi_{j, n}\right\}_{n=1}^{\infty}$ in $L_{E}$ such that $\psi_{j, n}$ converges to $b$ uniformly on compact subsets of $W_{j} \bigwedge_{E}^{n=1}$ and $|\nu|$-almost everywhere on $E$, and such that $\psi_{j, n}(x) \in T_{j}$ for all $x \in W_{j} \backslash E$ and $|\nu|$-almost all $x \in W_{j} \cap E$. Let $\left\{\phi_{1}, \cdots, \phi_{r}\right\}$ be a continuous partition of unity subordinate to $\left\{W_{1}, \cdots, W_{r}\right\}$, and set $\psi_{n}=\Sigma_{j=1}^{r} \phi_{j} \psi_{j n}$. Then it is easily verified that $\psi_{n} \in L_{E} \cap N^{\prime}$, and $\psi_{n}$ converges to $b$ in the $\tau$-topology.

To conclude the proof of Lemma 5.3, take $b \in \mathcal{F}$ with $\rho(b)=F$. Since $b$ belongs to the $\tau$-closure of $N^{\prime} \cap L_{E}$, and since $\operatorname{Re} \int g d \nu \leq a$ for all $g \in N^{\prime} \cap L_{E}$, we have $\operatorname{Re} \int b d \nu \leq a$. Since $\int b d \nu=\int b d \mu=\int F d \mu$, we have $\operatorname{Re} \int F d \mu \leq a$, and the proof is complete.

6. The distance estimate for arbitrary sets. In order to extend the results of the preceding section to nonclosed sets $E$, we must make a further assumption 
on $H$. We say that $H$ is pointwise boundedly closed if every pointwise limit on $U$ of a bounded sequence in $H$ also belongs to $H$. By the Krein-Schmulian theorem, this is equivalent to asserting that $H$ be weak-star closed in $L^{\infty}(\sigma)$, where $\sigma$ is the area measure on $U$. Our principal results in this section are the following.

6.1 Theorem. Let $H$ be a stable subalgebra of $H^{\infty}(U)$ which is pointwise boundedly closed. Let $E$ be a subset of $\partial U$ whicb is an $F_{\sigma^{-s e t}}$. Then the following are equivalent:

(i) $d\left(b, H_{E}\right)=d(b, H)$, for all $b \in L_{E}$.

(ii) $H_{E}$ is pointwise boundedly dense in $H$.

(iii) There is a sequence $\left\{E_{n}\right\}_{n=1}^{\infty}$ of closed subsets of $\partial U$ such that $E=\bigcup_{E_{n}}$, and $H_{E_{n}}$ is pointwise boundedly dense in $H$.

6.2 Theorem. Let $H$ be a stable subalgebra of $H^{\infty}(U)$ which is pointwise boundedly closed. Let $Q$ be a subset of $\partial U$ such that $Q$ is an $F_{\sigma^{-s e t}}$, and $H_{Q}$ is pointwise boundedly dense in $H$. If $E$ is an arbitrary subset of $Q$, then

$$
d\left(b, H_{E}\right)=d(b, H), \text { all } b \in L_{E} .
$$

First we prove a lemma.

6.3 Lemma. Let $H$ be a stable subalgebra of $H^{\infty}(U)$ which is pointwise boundedly closed, and let $E$ be a closed subset of $\partial U$ such that $H_{E}$ is pointwise boundedly dense in $H$. Suppose $T$ is a closed subset of $\partial U, b_{1}, \cdots, b_{k} \in L_{E \cup T}$ $X_{1}, \cdots, X_{k}$ are compact subsets of $\bar{U}$, and $\eta_{1}, \cdots, \eta_{k}$ are positive numbers. Let $f \in H_{T}$ and suppose $\left\|f-b_{i}\right\|_{V_{i} \cap U}<\eta_{i}$ for some neighborbood $V_{i}$ of $X_{i}$, $i=1, \cdots, k$. Let $\epsilon>0$ and let $S \subseteq U$ be at a positive distance from $E$. Then we can find $g \in H_{E \cup T}$, with $|g-f|<\epsilon$ on $S$ and $\left\|g-b_{i}\right\|_{V_{i}^{\prime} \cap U} \leq \eta_{i}$ for some neighborbood $V_{i}^{\prime}$ of $X_{i}, i=1, \ldots, k$.

Proof. First suppose $E \cap T=\varnothing$. Then the conclusion follows from Lemma 5.3, with $H_{T}$ in place of $H$ and $H_{T \cup E}$ in place of $B$. Lemma 5.2 guarantees that the hypotheses of Lemma 5.3 are fulfilled.

In the general case we put $E_{n}=\{z \in E: d(z, T) \geq 1 / n\}$. For $i=1, \cdots, k$ choose a compact neighborhood $M_{i}$ of $X_{i}$ contained in $V_{i}$. We define by induction on $n$, for $n=1,2, \ldots$, functions $g_{n} \in H_{E_{n} \cup T}$ and $b_{n}^{*} \in C(\bar{U})$, and compact neighborhoods $X_{n}^{*}$ of $E_{n} \cup T$ in $\bar{U}$, such that
(A) $\left|g_{1}-f\right|<\epsilon / 2$ and $\left|g_{n}-g_{n-1}\right|<\epsilon 2^{-n}$ on $S$.
(B) $\left\|g_{n}-b_{i}\right\|_{W_{n i} \cap U}<\eta_{i}$ for some neighborhood $W_{n i}$ of $M_{i}, i=1, \ldots, k$.
(C) $\left\|g_{n}-b_{k}^{*}\right\|_{W_{n k}^{*} \cap U}<1 / k$ for some neighborhood $W_{n k}^{*}$ of $X_{k}^{*}, k \leq n$. 
For the induction step, the case already treated yields $g_{n}$ satisfying everything except (C) for $k=n$; for that we let $b_{n}^{*}$ be any continuous extension of $\left.g_{n}\right|_{E_{n} \cup T}$ to $\vec{U}$ and then choose $X_{n}^{*}$ to satisfy (C).

Some subsequence of $\left\{g_{n}\right\}$ converges pointwise on $U$ to $g \in H$, which satisfies $|g-f|<\epsilon$ on $S,\left\|g-b_{i}\right\|_{M_{i} \cap U} \leq \eta_{i},\left\|g-b_{k}^{*}\right\|_{X_{k}^{*} \cap U}<1 / k$ for each $k$. The last condition implies $g \in H_{E \cup T}$, and the proof is complete.

Now to prove Theorem 6.1 , it suffices to show that (iii) implies (i). Hence both Theorems 6.1 and 6.2 will be proved, once we establish the following more general result.

6.4 Lemma. Let $H$ be a stable subalgebra of $H^{\infty}(U)$ which is pointwise boundedly closed. Let $\left\{E_{n}\right\}$ be a sequence of compact subsets of $\partial U$, such that eacb $H_{E_{n}}$ is pointwise boundedly dense in $H$. Let $E \subseteq \bigcup E_{n^{*}}$ Let $T$ be a compact subset of $\partial U$, let $f \in H_{T}$ and $b \in L_{E \cup T}$ satisfy $\|f-b\|<1$. Let $S$ be a subset of $U$ whose closure does not meet $E$ and let $\epsilon>0$. Then we can find $g \in H_{E \cup T}$ with $\|g-b\| \leq 1$, and $|g-f|<\epsilon$ on $S$.

Proof. We may assume $E \cap T=\varnothing$. First suppose $E=\bigcup_{n_{n}}$. Let $F_{\underline{n}}=$ $\bigcup_{i=1}^{n} E_{i}$. We construct by induction on $n$ functions $g_{n} \in H_{F_{n} \cup T^{\prime}} b_{n} \in C \stackrel{\underline{U}}{(U)}$, and compact neighborhoods $X_{n}$ of $E_{n} \cup T$ such that

(A) $\left|g_{1}-f\right|<\epsilon / 2$ and $\left|g_{n}-g_{n-1}\right|<\epsilon 2^{-n}$ on $S$.

(B) $\left\|g_{n}-b\right\|<1$.

(C) $\left\|g_{n}-b_{k}\right\|_{W_{n k} \cap U}<1 / k$ for some neighborhood $W_{n k}$ of $X_{k}, k \leq n$.

For the induction step Lemma 6.3 yields $g_{n}$ satisfying everything except (C) for $k=n$, and that case is dealt with as in the proof of Lemma 6.3.

Some subsequence of $\left\{g_{n}\right\}$ converges pointwise on $U$ to $g \in H$, and $g$ satisfies $|g-f|<\epsilon$ on $S,\|g-b\| \leq 1$, and $\left\|g-b_{k}\right\|_{X_{k} \cap U}<2 / k$ for each $k$. The last fact implies $g \in H_{E \cup T}$, and the proof is complete when $E=\bigcup E_{n}$.

In the general case, choose $\eta>0$ with $2 \eta<1-\|f-b\|$, and choose a neighborhood $V$ of $E$ not meeting $T \cup \bar{S}$ so that there exists $b^{\prime} \in L_{V \cup T}$ with $\left\|b^{\prime}-b\right\|<\eta$. The special case above applies to $F=V \cap\left(\bigcup E_{n}\right)$, so we can find $g \in L_{F \cup T}$ with $|g-f|<\epsilon$ on $S$ and $\left\|g-b^{\prime}\right\|<\|f-b\|+\eta$. Then $\|g-b\|<$ $\|f-b\|+2 \eta<1$, which proves the general case.

7. The algebras $\widetilde{H}_{E}$. In this section, we indicate how the techniques we have developed apply to another class of subalgebras of $H$. If $E$ is a subset of $\partial U$, we denote by $\widetilde{H}_{E}$ the algebra of uniform limits of those functions in $H_{E}$ which extend to be continuous in a neighborhood of $E$. If $E$ is a relatively open subset of $\partial U$, then $\widetilde{H}_{E}$ coincides with $H_{E}$. If $H$ is stable, then $\widetilde{H}_{E}$ is also stable. The following theorem is analogous to Theorem 5.1.

7.1 Theorem. Let $H$ be a closed stable subalgebra of $H^{\infty}(U)$, and let $E$ be a closed subset of $\partial U$. The following are equivalent: 
(i) $d\left(b, \widetilde{H}_{E}\right)=d(b, H)$, all $b \in L_{E}$.

(ii) $\widetilde{H}_{E}$ is pointwise boundedly dense in $H$.

(iii) $\widetilde{H}_{E}=H_{E}$, and $H_{E}$ is pointwise boundedly dense in $H$.

Proof. If (i) is true, then $d\left(b, \widetilde{H}_{E}\right)=0$ for all $b \in H_{E}$, so that $\widetilde{H}_{E}=H_{E}$. An application of Theorem 5.1 then shows that (i) and (iii) are equivalent, and that they imply (ii). It suffices then to show that (ii) implies (i). In order to do this, we note the following extension of Lemma 5.2.

7.2 Lemma. Let $H$ be a closed stable subalgebra of $H^{\infty}(U)$, and let $E$ be a closed subset of $\partial U$. Let $T$ be a closed subset of $\vec{U}$, and let $f \in H$ extend continuously to a neighborbood of T. Suppose there is a sequence $\left\{f_{n}\right\}$ in $\widetilde{H}_{E}$ converging pointwise to $f$ on $U$, sucb that $\left\|f_{n}\right\| \leq M$. Then there is a sequence $\left\{F_{n}\right\}$ in $H$ sucb that $\left\|F_{n}\right\| \leq 2 M, F_{n}$ extends continuously to a neighborbood of $E \cup T$, and $F_{n}$ converges to $f$ uniformly on $T$ and on eacb subset of $U$ at a positive distance from $E$.

Proof. We proceed exactly as in Lemma 5.2, except that instead of setting $g=0$ on a neighborhood of $E$ (now $E \cap T$ need not be empty), we take $g=0$ on a neighborhood of $E \backslash V$, where $V$ is a neighborhood of $T$ on which $f$ is continuous. Then $\psi_{n}$ will still be continuous on a neighborhood of $\dot{E}$, and the rest of the argument is as before.

Proof of 7.1 (concluded). That (ii) implies (i) now follows from Lemma 5.3, with $B=\widetilde{H}_{E}, k=1, b_{1}=b, X_{1}=\bar{U}$, and $\eta_{1}=d(b, H)+\epsilon$.

The theorems in $\$ 6$ can also be extended to cover the algebras $\widetilde{H}_{E}$. The analogue of Lemma 6.4 is the following.

7.3 Theorem. Let $H$ be a stable subalgebra of $H^{\infty}(U)$ which is pointwise boundedly closed. Let $\left\{E_{n}\right\}$ be a sequence of compact subsets of $\partial U$, such that each $\widetilde{H}_{E_{n}}$ is pointwise boundedly dense in $H$. Let $E \subseteq \bigcup E_{n^{*}}$. Let $T$ be a compact subset of $\partial U$, and let $f \in H$ extend continuously to a neigbborbood of $T$. Let $b \in L_{E \cup T}$ satisfy $\|f-b\|<1$, let $S$ be a subset of $U$ whose closure does not meet $E$, and let $\epsilon>0$. Then we can find $g \in \widetilde{H}_{E \cup T}$ with $\|g-b\| \leq 1$ and $|g-f|<\epsilon$ on $S$.

Proof. As before, we can assume $E=\bigcup E_{n}$. Using Lemmas 7.2 and 5.3 we construct inductively a compact neighborhood $M_{0}$ of $T$, compact neighborhoods $M_{n}$ of $F_{n}=E_{1} \cup \cdots \cup E_{n} \cup T$ and functions $f_{n} \in \widetilde{H}_{M \cap \partial U}$ such that $\left\|f_{n}-b\right\|<1$, $\left|f_{1}-f\right|<\epsilon / 2$ on $S$, and $\left|f_{n+1}-f_{n}\right|<\epsilon / 2^{n+1}$ on $S \cup M_{n}$. Then $f_{n} \rightarrow g$ say, pointwise on $U$ and uniformly on each $M_{n}$, so $g \in \widetilde{H}_{E}$ and $\|g-b\| \leq 1$.

We also state explicitly the analogues of Theorems 6.1 and 6.2 , since we will need the results later. 
7.4 Theorem. Let $H$ be a stable subalgebra of $H^{\infty}(U)$ which is pointwise boundedly closed. Let $E$ be a $\sigma$-compact subset of $\partial U$. Then the following are equivalent:

(i) $d\left(b, \widetilde{H}_{E}\right)=d(b, H)$, all $b \in L_{E}$.

(ii) $\widetilde{H}_{E}=H_{E}$, and $H_{E}$ is pointwise boundedly dense in $H$.

(iii) $\widetilde{H}_{E}$ is pointwise boundedly dense in $H$.

(iv) There is a sequence $\left\{E_{n}\right\}_{n=1}^{\infty}$ of closed subsets of $\partial U$ such that $E=\bigcup E_{n}$, and $\widetilde{H}_{E_{n}}$ is pointwise boundedly dense in $H$.

7.5 Theorem. Let $H$ be a stable subalgebra of $H^{\infty}(U)$ which is pointwise boundedly closed. Let $Q$ be a $\sigma$-compact subset of $\partial U$ sucb that $\widetilde{H}_{Q}$ is pointwise boundedly dense in $H$. If $E$ is any subset of $Q$, then $\widetilde{H}_{E}=H_{E}$, and $d\left(b, H_{E}\right)=d(b, H)$, all $b \in L_{E}$.

To prove 7.4 notice that (i) implies $\widetilde{H}_{E}=H_{E}$, so that (ii) holds by 6.1 . Clearly (ii) implies (iii) and (iii) implies (iv). But 7.3 shows that (iv) implies (i). To prove 7.5 , observe that $d\left(b, \widetilde{H}_{E}\right)=d(b, H)$ for all $b \in L_{E}$, by 7.3 , and that means $\widetilde{H}_{E}=H_{E}$.

8. Capacity conditions and distance estimates. In this section we treat specifically the case $H=H^{\infty}(U)$, and we seek geometric conditions on $U$ which imply the distance estimates. In light of the equivalences of distance estimates and bounded pointwise density, these conditions will be expressed using analytic capacity.

Let $Q$ be a subset of the complex plane C. The analytic capacity $\gamma(Q)$ is defined to be $\sup \left\{\left|f^{\prime}(\infty)\right|: f\right.$ is analytic off a compact subset of $Q,|f| \leq 1$, $f(\infty)=0\}$. The continuous analytic capacity $a(Q)$ is defined to be $\sup \left\{\left|f^{\prime}(\infty)\right|\right.$ : $f \in C\left(S^{2}\right), f$ is analytic off a compact subset of $\left.Q,|f| \leq 1, f(\infty)=0\right\}$. Evidently $\gamma$ and $\alpha$ are monotone set functions satisfying $\alpha(Q) \leq \gamma(Q)$, and $\alpha(Q)$ coincides with $\gamma(Q)$ whenever $Q$ is open. In general, $\gamma(Q) \leq$ diameter $Q$. If $Q$ is compact and connected, $\gamma(Q)$ is comparable to diameter:

$$
\gamma(Q) \leq \text { diameter } Q \leq 4 \gamma(Q), \quad Q \text { a continuum. }
$$

For proofs of these facts and a discussion of other elementary properties of analytic.capacity, see [10]. The approximation result which enables us to obtain distance estimates is the following.

8.1 Lemma. Let $J$ be a compact subset of $\partial U$ for which there exists $c>0, r \geq 1$ and $\delta_{0}>0$ satisfying $\gamma(\Delta(z ; \delta) \backslash U) \leq c \alpha(\Delta(z ; r \delta) \backslash U)$ for all $z \in J$ and $0<\delta<\delta_{0}$. Suppose $T$ is a compact subset of $\partial U$, and $f \in H^{\infty}(U)$ extends continuously to a neighborbood of $T$. Then there is a sequence $\left\{f_{n}\right\}$ in $H^{\infty}(U)$ such that each $f_{n}$ extends continuously to a neighborbood of $J \cup T, f_{n}$ converges 
to $f$ uniformly on $T$ and on eacb subset of $U$ at a positive distance from $J$, and $\left\|f_{n}\right\|_{U} \leq C\|f\|_{U}$, where the constant $C$ depends only on $c$ and $r$. If moreover $S$ is some prescribed compact subset of zero length lying on a $C^{2}$-curve, then we can in addition take each $f_{n}$ to be analytic in a neigbborbood of $S$.

We omit the proof, since it is a standard application of Vitushkin's techniques. For more general theorems of this sort, see [3], or [13].

8.2 Theorem. Let $E$ be a subset of $\partial U$. Let $S$ be a countable union of compact sets of zero length lying on $C^{2}$ curves. Suppose that for each $z \in E \backslash S$, there exist constants $c>0, r \geq 1$ and $\delta_{0}>0$ sucb that $\gamma(\Delta(z ; \delta) \backslash U) \leq$ $c \alpha(\Delta(z ; r \delta) \backslash U), 0<\delta \leq \delta_{0}$. Then

$$
d\left(b, H^{\infty}(U)\right)=d\left(b, \widetilde{H}_{E}^{\infty}(U)\right) \text {, all } b \in L_{E} .
$$

Proof. Write $S=\bigcup S_{n}$, where $S_{n}$ is a compact set of zero length, lying on a $C^{2}$ curve. Let

$$
Q_{n}=\{z \in \partial U: \gamma(\Delta(z ; \delta) \backslash U) \leq n \alpha(\Delta(z ; n \delta) \backslash U), 0<\delta<1 / n\}
$$

Then $Q_{n}$ is compact. By Lemma $8.1, \widetilde{H}_{Q_{n} \cup S_{n}}^{\infty}(U)$ is pointwise boundedly dense in $H^{\infty}(U)$. Applying Theorems 7.4 and 7.5, with $E_{n}=Q_{n} \cup S_{n}$, we obtain the desired distance estimate.

Theorem 8.2 shows in particular that every $f \in H_{E}^{\infty}(U)$ can be approximated uniformly on $U$ by functions in $H^{\infty}(U)$ which extend continuously to a neighborhood of $E$. Under the appropriate estimates on analytic capacity, these functions can in turn be approximated uniformly by functions which extend to be analytic in a neighborhood of E. In particular, if we apply Theorems 4.11 and 8.4 of [3], we obtain the following result.

8.3 Theorem. Let $E$ be a subset of $\partial U$. Let $S$ be a countable union of compact sets of zero length lying on $C^{2}$ curves. Suppose that for each $z \in E \backslash S$, there exist constants $c>0, r \geq 1$ and $\delta_{0}>0$ sucb that $\gamma(\Delta(z ; \delta) \backslash U) \leq$ $c \gamma(\Delta(z ; r \delta) \backslash \bar{U}), 0<\delta \leq \delta_{0}$. Then

$$
d\left(b, H^{\infty}(U)\right)=d\left(b, H_{E}^{\infty}(U)\right), \text { all } b \in L_{E} \cdot
$$

Moreover, every $f \in H_{E}^{\infty}(U)$ can be approximated uniformly on $U$ by functions which extend to be analytic on $E$.

The capacity estimate of Theorem 8.3 obtains at each point of $\partial U$ which lies on the boundary of a component of $\mathrm{C} \backslash \bar{U}$. The theorem applies, for instance, in the case that $U=\Delta$ is the open unit disc.

9. The algebras $H^{\infty}(\sigma)$ and $H^{\infty}(\mu)$. The Sarason-Zalcman results mentioned in the introduction give distance estimates for functions defined only on $\partial U$. 
On the other hand, we have been dealing with functions defined on $U$. In order to obtain a genuine extension of their theorems, we must reinterpret our work in terms of boundary functions. We do so in this section by introducing the stable algebras $H^{\infty}(\sigma)$ and $H^{\infty}(\mu)$, which are also of some interest in their own right.

Let $\sigma$ be the area measure $d x d y$ on $U$, and let $H^{\infty}(\sigma)$ denote the weak-star closure of $A(U)$ in $L^{\infty}(\sigma)$. The main theorem in [4] asserts the following.

9.1 Lemma. If $f \in H^{\infty}(\sigma)$, there is a sequence $f_{n} \in A(U)$ such that $\left\|f_{n}\right\| \leq$ $\|f\|$, and $f_{n}$ converges uniformly to $f$ on each compact subset of $U$.

In particular, the functions in $H^{\infty}(\sigma)$ are analytic on $U$, and $H^{\infty}(\sigma)$ is a stable subalgebra of $H^{\infty}(U)$. The algebras $H^{\infty}(\sigma)$ and $H^{\infty}(U)$ will coincide when and only when $A(U)$ is pointwise boundedly dense in $H^{\infty}(U)$. As a corollary of our previous efforts, we note the following theorem, which includes Theorem 1.2 and its Corollary 1.3.

9.2 Theorem. If $E$ is any subset of $\partial U$, then

$$
d\left(b, H^{\infty}(\sigma)\right)=d\left(b, H_{E}^{\infty}(\sigma)\right) \text {, all } b \in L_{E} .
$$

Every function in $H_{E}^{\infty}(\sigma)$ can be approximated uniformly by functions in $H^{\infty}(\sigma)$ which extend continuously to an open subset of $\partial U$ containing $E$.

Proof. The distance estimate follows from Theorem 6.2, with $Q=\partial U$. The second assertion follows from the distance estimate (cf. the proof of Corollary 1.3).

If $K$ is a compact subset of $\mathbf{C}$, then $R(K)$ is the algebra of continuous functions on $K$ which can be approximated uniformly on $K$ by rational functions with poles off $K$. Consequently $R(\bar{U})$ is a subalgebra of $A(U)$. If $R(\bar{U})$ coincides with $\Lambda(U)$, then Vitushkin's constructive techniques can be used to show (cf. [3]) that every function $f \in H^{\infty}(U)$ which extends to be continuous on a subset $E$ of $\partial U$ can be approximated uniformly by functions in $H^{\infty}(U)$ which are analytic on $E$. In fact, the approximators can be chosen to belong to any stable subalgebra of $H^{\infty}(U)$ to which $f$ belongs. Consequently we obtain the following corollary to 9.2 .

9.3 Corollary. Suppose that $\Lambda(U)=R(\bar{U})$. If $E$ is any subset of $\partial U$, and $f \in H^{\infty}(\sigma)$ extends continuously to each point of $E$, then $f$ can be approximated uniformly on $U$ by functions which are analytic on $E$.

Let $\mu_{z}$ be the harmonic measure on $\partial U$ for the point $z \in U$. Let $\mu$ be any positive measure on $\partial U$ such that $\mu(E)=0$ if and only if $\mu_{z}(E)=0$ for all $z \in U$; for instance, we can choose one point $z$ from each component of $U$, and set $\mu=\Sigma \mu_{j} / 2^{j}$. If $f \in L^{\infty}(\mu)$, we define $\tilde{f}(z)=\int f d \mu_{z}, z \in U$. Then $\tilde{f}$ 
is evidently a bounded harmonic function on $U$, satisfying $\|\widetilde{T}\|_{U} \leq\|f\|_{\infty}$.

9.4 Lemma. The map $f \rightarrow \widetilde{f}$ is a linear isometric isomorphism of $L^{\infty}(\mu)$ and a weak-star closed subspace of. $L^{\infty}(\sigma)$. The map is bicontinuous when $L^{\infty}(\mu)$ and $L^{\infty}(\sigma)$ are given their weak-star topologies.

Proof. The isometry part is an elementary result in potential theory (cf. [2, Lemma 2.2]). It follows easily that a bounded net $\left\{f_{\alpha}\right\}$ in $L^{\infty}(\mu)$ converges weak-star in $L^{\infty}(\mu)$ if and only if $\left\{\tilde{f}_{a}\right\}$ converges weak-star in $L^{\infty}(\sigma)$. These facts, together with the Krein-Schmulian theorem, yield the remaining conclusions of the lemma.

9.5 Lemma. Suppose $\lambda_{0} \in \partial U$ and $f \in L^{\infty}(\mu)$. Then

$$
\underset{\partial U \ni \lambda \rightarrow \lambda_{0}}{\text { ess } \lim \sup _{\partial}}|f(\lambda)| \leq \lim _{U \ni z \rightarrow \lambda_{0}} \sup _{0} \tilde{f}(z) \mid .
$$

If $\lambda_{0}$ is a regular boundary point of $U$, or if $\tilde{f}$ is analytic on $U$, then equality bolds.

Proof. The first inequality follows easily by applying Lemma 9.4 to $U \cap \Delta\left(\lambda_{0} ; \delta\right)$ for small $\delta>0$. If $\lambda_{0}$ is a regular boundary point, then $\mu_{z}$ clusters at the point mass at $\lambda_{0}$ as $z \rightarrow \lambda_{0}$, so we easily get equality here. If $\widetilde{f}$ is analytic, then we can apply Iversen's theorem to get equality (cf. [9]).

Now let $H^{\infty}(\mu)$ be the weak-star closure of $A(U)$ in $L^{\infty}(\mu)$. In view of the preceding lemmas, we obtain the following.

9.6 Lemma. The map $f \rightarrow \tilde{f}$ is an isometric algebra isomorphism of $H^{\infty}(\mu)$ onto $H^{\infty}(\sigma)$. It is weak-star bicontinuous. If $f \in H^{\infty}(\mu)$ and $\lambda \in \partial U$, then $f$ is continuous at $\lambda$ if and only if $\tilde{f}$ is continuous at $\lambda$.

Notice that when $A(U)$ is pointwise boundedly dense in $H^{\infty}(U)$, the above lemma implies that the algebras $H^{\infty}(\mu), H^{\infty}(\sigma)$ and $H^{\infty}(U)$ all coincide. This occurs, for example, in the cases considered by Zalcman [20].

Suppose that $E$ is a subset of $\partial U$. Let $L_{E}^{\infty}(\mu)$ be the subspace of functions in $L^{\infty}(\mu)$ which are (essentially) continuous at every point of $E$. Then $f \in L_{E}^{\infty}(\mu)$ if and only if $f$ is constant on the "fiber" over each point of $E$ of the maximal ideal space of $L^{\infty}(\mu)$. Any function in $L_{E}^{\infty}(\mu)$ can be approximated uniformly by functions in $L_{E}^{\infty}(\mu)$ which are continuous on an open set containing $E$.

As before, we define $H_{E}^{\infty}(\mu)=L_{E}^{\infty}(\mu) \cap H^{\infty}(\mu)$. In view of Lemma 9.6, we see that $f \in H_{E}^{\infty}(\mu)$ if and only if $\widetilde{f} \in H_{E}^{\infty}(\sigma)$. Our main result is the following.

9.7 Theorem. Let $\mu$ be barmonic measure on $\partial U$ for $U$, and let $H^{\infty}(\mu)$ be the weak-star closure of $A(U)$ in $L^{\infty}(\mu)$. If $E$ is any subset of $\partial U$, then 


$$
d\left(b, H^{\infty}(\mu)\right)=d\left(b, H_{E}^{\infty}(\mu)\right), \text { all } b \in L_{E}^{\infty}(\mu) .
$$

Proof. It suffices to prove the theorem for functions $b \in L_{E}^{\infty}(\mu)$ which are continuous on an open subset of $\mathbf{C}$ containing $E$. Let $f \in H^{\infty}(\mu)$, and let $\epsilon>0$. For each $\lambda \in E$, lim $\sup _{\partial U \ni w \rightarrow \lambda}|f(w)-b(\lambda)| \leq\|f-b\|$. By Lemma 9.5,

$$
\lim _{U \ni z \rightarrow \lambda} \sup _{z \rightarrow}|\tilde{f}(z)-b(\lambda)| \leq\|f-b\| \text {. }
$$

It follows that $|\widetilde{f}(z)-b(z)|<\|f-b\|+\epsilon$ on some relatively open subset $V$ of $\bar{U}$ containing $E$. Construct an open subset $W$ of $U$ such that $W \cup V \supset U, U \cap \partial W \subset$ $V$, and $U \cap \partial W$ consists of a family of smooth curves. Define the continuous function $g$ on $W$ to be the harmonic extension of $\left.b\right|_{\partial W}$ to $W$. Since every point of $U \cap \partial W$ is regular, $g$ assumes the continuous boundary values $b$ on $U \cap \partial W$. Hence we can extend $g$ continuously to $U$ by declaring $g=b$ on $U \backslash W$. Then $g \in L_{E}(U)$, and $|g-\tilde{f}|<\|f-b\|+\epsilon$ on $U \backslash W$. In $W, g-\tilde{f}$ is then the harmonic extension of the function equal to $g-\tilde{f}$ on $U \cap \partial W$. It follows that $|g-\tilde{f}|<$ $\|f-b\|+\epsilon$ also on $W$, and thus $\|g-\tilde{f}\| \leq\|f-b\|+\epsilon$. Hence

$$
d\left(g, H^{\infty}(\sigma)\right) \leq\|f+b\|+\epsilon .
$$

By Theorem 9.2 there is a function $F \in H_{E}^{\infty}(\mu)$ such that $\|g-\widetilde{F}\| \leq\|f-b\|+2 \epsilon$. It follows easily that $|b-F| \leq\|f-b\|+2 \epsilon$, so that $d\left(b, H_{E}^{\infty}(\mu)\right)<\|f-b\|+2 \epsilon$. Letting $\epsilon \rightarrow 0$, and then taking the infimum over $f \in H^{\infty}(\mu)$, we obtain $d\left(b, H_{E}^{\infty}(\mu)\right) \leq d\left(b, H^{\infty}(\mu)\right)$. The reverse inequality is trivial.

Of course the above proof is much easier when $E$ is a subset of the regular points of $\partial U$. For then $b \in L_{E}^{\infty}(\mu)$ implies $\widetilde{b} \in L_{E}$, so that, by $9.2, d\left(\widetilde{b}, H_{E}^{\infty}(\sigma)\right)=$ $d\left(\widetilde{b}, H^{\infty}(\sigma)\right)$. Then since $f \leftrightarrow \widetilde{f}$ is an isometry, $d\left(\widetilde{b}, H^{\infty}(\sigma)\right)=d\left(b, H^{\infty}(\mu)\right)$, and $d\left(\widetilde{b}, H_{E}^{\infty}(\sigma)\right)=d\left(b, H_{E}^{\infty}(\mu)\right)$.

Again we obtain an approximation result as a corollary.

9.8 Corollary. If $E$ is an arbitrary subset of $\partial U$, and $f \in H_{E}^{\infty}(\mu)$, then $f$ can be approximated uniformly by functions in $H^{\infty}(\mu)$ which extend continuously to a neighborbood of $E$. If furthermore $A(U)=R(\bar{U})$, then every $f \in H_{E}^{\infty}(\mu)$ can be approximated uniformly by functions in $H^{\infty}(\mu)$ which are analytic on $E$.

Theorem 9.7 includes the Sarason-Zalcman theorems. Indeed, if we take $E=\partial U$, we obtain $d(b, A(U))=d\left(b, H^{\infty}(\mu)\right)$, all $b \in C(\partial U)$. This result has been extended to a uniform algebraic setting in [15].

10. A related distance estimate. In this section, we will treat the problem of estimating the distance from $f \in H$ to $H_{E}$. This is related to the preceding work by the following lemma.

10.1 Lemma. Let $H$ be a closed subalgebra of $H^{\infty}(U)$, and let $E$ be a subset of $\partial U$. Then the following assertions are equivalent: 
(i) There is a constant $c>0$ such that $d\left(b, H_{E}\right) \leq c d(b, H)$, all $b \in L_{E}$.

(ii) There is a constant $c^{\prime}>0$ such that $d\left(f, H_{E}\right) \leq c^{\prime} d\left(f, L_{E}\right)$, all $f \in H$.

(iii) $H+L_{E}$ is a closed subspace of $L(U)$.

Moreover, if $c$ and $c^{\prime}$ are the best possible constants in (i) and (ii) respectively, then $c-1 \leq c^{\prime} \leq c+1$.

Proof. The equivalence of (i) and (ii) is a simple consequence of the triangle inequality. Suppose for instance that (i) is true. Let $f \in H$, and let $\epsilon>0$. Choose $b \in L_{E}$ such that $\|f-b\|<d\left(f, L_{E}\right)+\epsilon$. Then $d\left(b, H_{E}\right) \leq c d(b, H)<$ $c d\left(f, L_{E}\right)+c \epsilon$, so there is $g \in H_{E}$ satisfying $\|b-g\|<c d\left(f, L_{E}\right)+c \epsilon$. Hence $d\left(f ; H_{E}\right) \leq\|f-g\| \leq\|f-b\|+\|b-g\| \leq(c+1)\left[d\left(f, L_{E}\right)+\epsilon\right]$. Letting $\epsilon \rightarrow 0$, we obtain the estimate (ii), with $c^{\prime}=c+1$. The proof that (ii) implies (i) is similar.

Since $H \cap L_{E}=H_{E}$, the natural mapping $L_{E} \rightarrow L(U) \rightarrow L(U) / H$ induces a mapping $\tau: L_{E} / H_{E} \rightarrow L(U) / H$. Then there is a constant $c$ such that $d\left(b, H_{E}\right) \leq$ $c d(b, H)$, all $b \in L_{E}$, if and only if the range $L_{E} / H$ of $\tau$ is closed. Since $H+L_{E}$ is the pre-image of $L_{E} / H$ under the quotient map $L(U) \rightarrow L(U) / H$, $L_{E} / H$ is closed if and only if $H+L_{E}$ is closed. That proves that (i) and (iii) are equivalent.

10.2 Corollary. If $d\left(b, H_{E}\right)=d(b, H)$ for all $b \in L_{E}$, then $d\left(f, H_{E}^{\infty}\right) \leq$ $2 d\left(f, L_{E}\right)$, all $f \in H$.

In the next two theorems we give instances in which this estimate can be improved upon.

10.3 Theorem. Let $H$ be a closed subalgebra of $H^{\infty}(U)$. Suppose that $E$ is a closed subset of $\partial U$ which is a peak interpolation set for $H_{E}$. Then $d\left(b, H_{E}\right)=$ $d(b, H)$, all $b \in L_{E}$, and $d\left(f, H_{E}\right)=d\left(f, L_{E}\right)$, all $f \in H$.

Proof. Let $f \in H$ and $b \in L_{E}$ satisfy $\|f-b\| \leq 1$. Let $\epsilon>0$. It suffices to find $g \in H_{E}$ with $\|f-g\| \leq 1+\epsilon$ and $\|b-g\| \leq 1+\epsilon$.

Since $E$ is an interpolation set for $H_{E}$, we can find $\widetilde{b} \in H_{E}$ such that $\widetilde{b}=b$ on $E$. Replacing $f$ and $b$ by $f-\widetilde{b}$ and $b-\widetilde{b}$, we may assume $b=0$ on $E$.

Choose an open neighborhood $V$ of $E$ in $\bar{U}$ such that $|b|<\epsilon / 2$, and hence $|f|<1+\epsilon / 2$, on $V \cap U$. Since $E$ is a peak set for $H_{E}$, we can find $F \in H_{E}$ with $F=1$ on $E,\|F\| \leq 1,\|1-F\| \leq 1$, and $|F|<\epsilon /\|f\|$ on $U \backslash V$. Set $g=(1-F) f \epsilon H_{E}$. Then $g-f=F f$ is bounded by $1+\epsilon / 2$ on $V$, and it is bounded by $\epsilon$ on $U \backslash V$, so that $\|g-f\|<1+\epsilon / 2$. Since $|g| \leq|1-F||f|<1+\epsilon / 2$ on $V, g-b$ is bounded by $|g|+|b|<1+\epsilon$ on $V$. Also, $g-b=f-b-F f$ is bounded by $|f-b|+|F f|<1+\epsilon$ on $U \backslash V$, so that $\|g-b\|<1+\epsilon$. That proves the theorem. 
10.4 Theorem. Let $H$ be a closed subalgebra of $H^{\infty}(U)$. Let $E$ be a closed subset of $\partial U$ such that $d\left(b, H_{E}\right)=d(b, H)$ for all $b \in L_{E}$, and such that the restriction $\left.H_{E}\right|_{E}$ is dense in $C(E)$. If $f \in H$ but $f \notin H_{E}$, then $d\left(f, H_{E}\right)<$ $2 d\left(f, L_{E}\right)$.

Proof. We will use the notation introduced in $\$ 2$. Suppose $f \in H$ does not belong to $H_{E}$. By the Hahn-Banach theorem, there is a measure $\eta$ on $M$ such that $\|\eta\|=1, \eta \perp H_{E}$, and

$$
d\left(f, H_{E}\right)=\int f d \eta \neq 0 .
$$

Then $\pi(\eta) \in\left(H_{E}\right)^{\perp}$. By Lemma 2.1 , there is a measure $\mu$ on $\mathbb{M}$ such that $\mu \perp H$, $\pi(\mu)=\pi(\eta)$, and $\|\mu\|=\|\pi(\eta)\|$. Then $\eta-\mu \perp L_{E}$, so that

$$
d\left(f, H_{E}\right)=\int f d(\eta-\mu) \leq\|\eta-\mu\| d\left(f, L_{E}\right) .
$$

So it suffices to show that $\|\eta-\mu\|<2$.

Now $\|\pi(\mu)\| \leq 1$, and $\pi(\mu) \perp H_{E}$. By our hypothesis, $E$ can carry no measures orthogonal to $H_{E}$, so that $\pi(\mu)$ cannot be supported on $E$. Hence

$$
1>|(\pi \mu)(E)|=|\mu|\left(\pi^{-1}(E)\right) .
$$

Since $\pi$ is one-to-one off $\pi^{-1}(E), \mu$ and $\eta$ coincide on $M \backslash \pi^{-1}(E)$. Hence $\|\mu-\eta\|=|\mu-\eta|\left(\pi^{-1}(E)\right) \leq|\mu|\left(\pi^{-1}(E)\right)+|\eta|\left(\pi^{-1}(E)\right)<1+\|\eta\| \leq 2$, as required.

The remainder of this section is devoted to showing that the constant 2 in Theorems 10.2 and 10.4 cannot be improved upon. We will be working on the unit disc $\Delta$.

In [1], Adamyan, Arov and Krein give an example of a function $v \in C(\partial \Delta)$ which has no nearest element in $A(\Delta)$. Their example can be used to show that Theorems 10.2 and 10.4 are sharp. The example goes as follows (we thank D. Sarason for pointing out the existence of the example and the proof which follows).

Let $u$ be a continuous function on $\partial \Delta$ such that the harmonic conjugate ${ }^{*} u$ of $u$ is not continuous. Then the function

$$
b(z)=\bar{z} e^{i^{*} u}=\left(\bar{z} e^{-u}\right) e^{u+i^{*} u}
$$

belongs to $H^{\infty}(d \theta)+C(\partial \Delta)$, because the latter space is an algebra [17]. Hence we can write $b=f+v$, where $f \in H^{\infty}(d \theta)$ and $v \in C(\partial \Delta)$. Then $d(\nu, A(\Delta))=$ $d\left(\nu, H^{\infty}\right) \leq\|b\|=1$.

Suppose there is $g \in A$ such that $\|v-g\| \leq 1$. Let $F=z e^{-u-i^{*} u} \in H_{0}^{1}$. Then

$$
\int|F| d \theta=\int b F d \theta=\int v F d \theta=\int(v-g) F d \theta
$$


so that $|F|=(v-g) F$ a.e. Substituting the expression for $F$, we obtain $e^{u+i^{*} u}=e^{u}(v-g) z \in H^{\infty} \cap C=A$, which contradicts the fact that ${ }^{*} u$ is discontinuous. We conclude that $d(v, A(\Delta))=1$, and that there is no nearest point to $v$ in $A(\Delta)$.

Now suppose that $u$ is chosen so that the cluster set of $e^{i^{*} u}$ at each point of $\partial \Delta$ is the whole unit circle. We claim that $d(f, A(\Delta))=2$, and that there is no nearest point to $f$ in $A(\Delta)$. Indeed, since $d(f, C) \leq\|b\| \leq 1$, we have $d(f, A(\Delta)) \leq 2$. If $g \in A(\Delta)$ is arbitrary, then $\|g-v\|>1$. Since $g-v$ is continuous, while the cluster set of $b$ at every point of $\partial \Delta$ includes the whole unit circle, we have $\|b+g-v\|=\|f+g\|>2$. Hence $d(f, A(\Delta))=2$, and from 10.2 we have $d(f, C)=1$.

10.5 Theorem. There exists $/ \in H^{\infty}(\Delta)$ such that $d(f, C(\bar{\Delta}))=1$, and $d(f, A(\Delta))=2$.

That shows that 10.2 is sharp. To show that 10.4 is sharp, we modify the above construction.

10.6 Theorem. If $E$ is a subset of $\partial \Delta$ of positive inner length, and if $\epsilon>0$, then there is $f \in H^{\infty}(\Delta)$ such that $d(f, C(\bar{\Delta})) \leq 1$, while $d\left(f, H_{E}^{\infty}(\Delta)\right)>2-\epsilon$.

Proof. By shrinking $E$, we can assume that $E$ is a closed proper subset of $\partial \Delta$ which has positive length. Let $u$ be a positive continuous function on $\partial \Delta$ such that $e^{i *} u$ has cluster set the unit circle at each point of $E$ and such that

$$
\int_{\partial \Delta \backslash E} e^{-u} d \theta<\epsilon / 2, \quad \int_{E} e^{-u} d \theta=1 .
$$

As above, set $b(z)=\bar{z} e^{i *} u=f+v$, where $f \in H^{\infty}$ and $v \in C$. Then $d(f, C) \leq$ $\|f+v\|=1$. Suppose that $g \in H_{E}^{\infty}$ satisfies $\|f-g\| \leq 2$. Then $\|v-g\| \leq 3$. Setting $F=e^{-u-i^{*} u} \in H_{0}^{1}$, we obtain $1+\epsilon / 2<\int e^{-u} d \theta=\int|F| d \theta=\int F(f+v) d \theta=$ $\int F(v-g) d \theta=\int_{\partial \Delta} \backslash E+\int_{E} \leq 3 \epsilon / 2+\|v-g\|_{E}$, so that $1-\epsilon<\|v-g\|_{E}$. Since $v-g$ is continuous on $E$, and the cluster set of $f+v$ at each point of $E$ includes the full unit circle, we obtain $2-\epsilon<\|f-g\|_{E}$. Hence $d\left(f, H_{E}^{\infty}\right) \geq 2-\epsilon$.

11. On $H+L_{E}$. Recall that $\mathbb{N}$ is the Stone-Čech compactification of $U$, so that $L \cong C(\mathbb{N})$. The inclusion $A(U) \hookrightarrow L$ induces a projection $\mathbb{M} \rightarrow \vec{U}$, which coincides with the coordinate function $Z$, regarded as an element of $C(\pi)$. For each $\lambda \in \bar{U}$, define the fiber $M_{\lambda}$ to be

$$
\mathbb{M}_{\lambda}=Z^{-1}(\lambda)=\{q \in \mathbb{M}: Z(q)=\lambda\} .
$$

Then $\mathbb{M}_{\lambda}=\{\lambda\}$ if $\lambda \in U$, while $\mathbb{M}_{\lambda}$ is quite complicated when $\lambda \in \partial U$. With this notation, 


$$
L_{E}=\left\{b \in C(\mathbb{T}):\left.b\right|_{\aleph_{\lambda}} \text { is constant for each } \lambda \in E\right\} .
$$

It follows that

$$
H+L_{E} \subseteq\left\{f \in C(\mathbb{M}):\left.\left.f\right|_{\boldsymbol{M}_{\lambda}} \in H\right|_{\pi_{\lambda}} \text { for all } \lambda \in E\right\} .
$$

If $H$ is a closed stable subalgebra of $H^{\infty}(U)$, and $\lambda \in \vec{U}$, then $\left.H\right|_{\mathbb{R}_{\lambda}}$ is a closed subalgebra of $C\left(\pi_{\lambda}\right)$ [cf. Theorem 3.5 and proof]. The set appearing at the right-hand side of the above inclusion is then a closed subalgebra of $C(\pi)$, but $H+L_{E}$ is a priori neither closed nor an algebra. In this section, we will show that under appropriate hypotheses, the above inclusion becomes an equality.

11.1 Lemma. Let $H$ be a closed stable subalgebra of $H^{\infty}(U)$, let $E$ be an arbitrary subset of $\partial U$, and let $\left[H+L_{E}\right]$ be the closure of $H+L_{E}$ in $C(\pi)$. Then

$$
\left[H+L_{E}\right]=\left\{g \in C(\Re):\left.\left.g\right|_{\boldsymbol{M}_{\lambda}} \in\left[H+L_{E}\right]\right|_{\boldsymbol{M}_{\lambda}}, \lambda \in \bar{E}\right\}
$$

Proof. Let $B$ be the restriction of $\left[H^{\infty}+L_{E}\right]$ to $Z^{-1}(\vec{E})$. Since $L_{E}$ includes all the functions in $C(M)$ which vanish on $Z^{-1}(\bar{E}), B$ is a closed subspace of $C\left(Z^{-1}(\bar{E})\right)$, and $\left[H^{\infty}+L_{E}\right]$ consists of the function in $B$, extended in all possible continuous ways to $M$.

Now note that $B$ is a $C(\bar{E})$-module. In fact, if $f \in H^{\infty}$ and $g$ is any rational functions with poles off $\partial U$, it is easy to see that $f g \in H^{\infty}+C(\partial U) \subseteq H^{\infty}+L_{E}$. Hence $B$ is an $R(\partial U)$-module. Since $B$ is also an $A(U)$-module, and since $A(U)+R(\partial U)$ is dense in $C(\partial U), B$ is a $C(\bar{E})$-module. By the theory of antisymmetric sets for uniform algebras (cf. $[10$, p. 61]), $B$ coincides with the functions in $C\left(Z^{-1}(\bar{E})\right)$ which belong to $B$ on each level set of $C(\bar{E})$. Since the level sets of $C(\bar{E})$, regarded as a subalgebra of $C\left(Z^{-1}(\bar{E})\right)$, are precisely the level sets of $Z$, we find that

$$
B=\left\{f \in C\left(Z^{-1}(\bar{E})\right):\left.\left.f\right|_{\boldsymbol{M}_{\lambda}} \in B\right|_{\boldsymbol{M}_{\lambda}}, \lambda \in \bar{E}\right\} .
$$

That proves the lemma.

11.2 Theorem. Let $H$ be a stable closed subalgebra of $H^{\infty}(U)$, and let $E$ be a closed subset of $\partial U$. Suppose there is a constant $c>0$ such that $d\left(b, H_{E}\right) \leq c d(b, H)$, all $b \in L_{E}$. Then $H+L_{E}$ is a closed subalgebra of $L(U)$ consisting of precisely the functions $f \in L(U)$ such that $\left.\left.f\right|_{\mathbb{N}_{\lambda}} \in H\right|_{\mathbb{\pi}_{\lambda}}$ for all $\lambda \in E$.

Proof. By Lemma 10.1, $H+L_{E}$ is a closed subspace of $L(U)$. Since the functions in $L_{E}$ are constant on each fiber over $\lambda \in E$, we have $\left.\left(H+L_{E}\right)\right|_{\pi_{\lambda}}=$ $\left.H\right|_{\pi_{\lambda}}, \lambda \in E$. Since $E$ is closed, $\left.L_{E}\right|_{\pi_{\lambda}}=C\left(\pi_{\lambda}\right)$ for $\lambda \notin E$. The desired 
description follows now from Lemma 10.1. In particular, from its explicit description it follows that $L_{E}+H$ is an algebra.

Next we wish to extend Theorem 11.2 to some classes of nonclosed sets. First we establish a lemma.

11.3 Lemma. Let $H$ be a stable closed subalgebra of $H^{\infty}(U)$. Suppose $S$ and $T$ are compact subsets of $\partial U$, and $T_{0}$ is a compact subset of the relative interior of $T$ in $\partial U$. If $f \in H+L_{S}$ and $f \in H+L_{T}$, then $f \in H+L_{S \cup T_{0}}$.

Proof. Let $f=f_{1}+b_{1}=f_{2}+b_{2}$, when $f_{j} \in H, b_{1} \in L_{S}$, and $b_{2} \in L_{T}$. Then $F=f_{1}-f_{2}=b_{2}-b_{1}$ belongs to $H_{T \cap S^{\circ}}$ Let $g$ be a smooth function with compact support, such that $g=0$ in a neighborhood of $T_{0}$, and $g=1$ in a neighborhood of S $\backslash T$. Applying the operator $T_{g}$ discussed in $\$ 3$, we obtain a function $F^{\prime}=T_{g}(F)$, which belong to ${ }^{H} T_{0}$. Since $\stackrel{g}{F}-F^{\prime}$ is continuous at the points of continuity of $F$, and at the points on the interior of the level set $\{g=1\}, F-F^{\prime} \in H_{S}$. Now $b_{2}-F^{\prime} \in L_{T_{0}}$, while on the other hand $b_{2}-F^{\prime}=b_{1}+\left(F-F^{\prime}\right) \in L_{S}$, so that $b_{2}-F^{\prime} \in L_{S \cup T_{0}} \cdot$ Hence

$$
f=\left(f_{2}+F^{\prime}\right)+\left(b_{2}-F^{\prime}\right) \in H+L_{S \cup T_{0}} \cdot
$$

11.4 Theorem. Let $H$ be a stable subalgebra of $H^{\infty}(U)$ which is pointwise boundedly closed. Suppose $Q$ is a o-compact subset of $\partial U$ sucb that $d\left(b, \widetilde{H}_{Q}\right)=$ $d(b, H)$ for all $b \in L_{Q}$. Then $H+L_{Q}$ is a closed subalgebra of $L(U)$, consisting of precisely the functions $f \in L(U)$ such that $\left.\left.f\right|_{\pi_{\lambda}} \in H\right|_{\pi_{\lambda}}$ for all $\lambda \in Q$.

Proof. By Lemma $10.1, H+L_{Q}$ is a closed subspace of $L(U)$. The family of $f$ 's described in 11.4 is a closed subalgebra of $L(U)$ which contains $H+L_{Q}$. To complete the proof, it will suffice to show that every such $f$ can be approximated uniformly by functions in $H+L_{Q}$.

Suppose then that $\epsilon>0$, and that $f \in L(U)$ satisfies $\|f\|<1$ and $\left.\left.f\right|_{\pi_{\lambda}} \in H\right|_{\pi_{\lambda}}$ for all $\lambda \in Q$. Write $Q$ as an increasing union of compact subsets $Q_{n}$ with $Q_{0}$ empty. By Theorem 11.2, $f \in H+L_{Q_{n}}$ for each $n$.

We will construct inductively sequences $\left\{f_{n}\right\},\left\{g_{n}\right\},\left\{b_{n}\right\}$ and $\left\{R_{n}\right\}$ such that

(i) $R_{0}$ is empty, $f_{0}=b_{0}=f$, and $g_{0}=0$.

(ii) $R_{n} \subseteq R_{n+1} \subseteq \bar{U}$, and $R_{n}$ is a closed neighborhood in $\bar{U}$ of $Q_{n} \cup\{z \in U$ : $d(z, \partial U) \geq 1 / n\}$.

(iii) $b_{n} \in L(U)$ extends continuously to a neighborhood of $R_{n}$.

(iv) $\left\|b_{n}\right\|<1$, and $b_{n} \in H+L_{Q_{j}}$ for all $j$.

(v) $\left|b_{n+1}-b_{n}\right|<1 / 2^{n}$ on $R_{n}$.

(vi) $g_{n} \in H, f_{n}=g_{n}+b_{n}$.

(vii) $\left\|f_{n+1}-f_{n}\right\|<\epsilon / 2^{n+1}$.

The induction step goes as follows. 
Suppose $f_{n}, g_{n}, b_{n}$ and $R_{n}$ have been constructed. Let $T$ be a compact neighborhood of $R_{n}$ in $\vec{U}$ such that $b_{n}$ extends continuously to a neighborhood $T^{\prime}$ of $T$. Then $f_{n} \in H+L_{T}$ and $f_{n} \in H+L_{Q_{n+1}}$. Using Lemma 11.3, we can write $f_{n}=g+b$, where $g \in H$ and $b \in L_{Q_{n+1} \cup T}$ extends continuously to a neighborhood of $T$. Set $F=g-g_{n}=b_{n}-b \in \widetilde{H}_{T}$, and note that $\|F+b\|=$ $\left\|b_{n}\right\|<1$. Now we apply Theorem 7.3 with $S=R_{n}, E=Q_{n+1} \backslash T$ and $T$. The hypothesis of that result holds because $E$ is $\sigma$-compact and $\widetilde{H}_{Q_{n+1}}$ is pointwise boundedly dense in $H$. By 7.3 there is a function $F^{\prime} \in \widetilde{H}_{Q_{n+1} \cup T}$ such that $\left\|F^{\prime}+b\right\|<1$ and $\left|F^{\prime}-F\right|<1 / 2^{n+1}$ on $R_{n}$. Set $q_{n+1}=g-F^{\prime} \in H$, and set $b_{n+1}^{*}=b+F^{\prime}$. Noting that $b_{n+1}^{*}-b_{n}=F^{\prime}-F$, we obtain

(iii) ${ }^{\prime} b_{n+1}^{*} \in L_{Q_{n+1} \cup T^{*}}$

(iv) $\left\|b_{n+1}^{*}\right\|<1$, and $b_{n+1}^{*} \in H+L_{Q_{j}}$ for all $j$.

(v) $\left|b_{n+1}^{*}-b_{n}\right|<1 / 2^{n+1}$ on $R_{n}$.

(vi) $f_{n}=g_{n+1}+b_{n+1}^{*}$.

Choose $u \in C(\bar{U})$ such that $u-b_{n+1}^{*}=0$ on $Q_{n+1} \cup T$. For $\delta>0$, choose $v_{\delta} \in C(\bar{U})$ such that $0 \leq v_{\delta} \leq 1, v_{\delta}=0$ on $Q_{n+1} \cup T$, and $v_{\delta}=1$ off a $\delta$-neighborhood of $Q_{n+1} \cup T$. Using Theorem 11.2, we see that $v_{\delta}\left(b_{n+1}^{*}-u\right)+$ $u \in H+L_{Q_{j}}$ for all $j$. Moreover, $v_{\delta}\left(b_{n+1}^{*}-u\right)+u$ extends continuously to a neighborhood of $T \cup Q_{n+1}$ in $\bar{U}$, and $v_{\delta}\left(b_{n+1}^{*}-u\right)+u$ converges uniformly to $b_{n+1}^{*}$ as $\delta \rightarrow 0$. If we set $b_{n+1}=v_{\delta}\left(b_{n+1}^{*}-u\right)+u$, for $\delta$ sufficiently small, then (iv) through (vii) will be valid, in view of (iv) ${ }^{\prime},(v)^{\prime}$ and (vi)'. On account of the continuity property of $b_{n+1}$, we can easily choose $R_{n+1}$ to satisfy (ii) and (iii). That completes the induction.

Now the $g_{n} \in H$ are converging pointwise boundedly to a function $g^{*} \in H^{\infty}(U)$. Since $H$ is assumed to be weak-star closed, $g^{*} \in H$. Moreover, $b_{n}$ converges to some function $b^{*} \in L(U)$, uniformly on a neighborhood of each $Q_{n}$, so that $b^{*} \in L_{Q}$. Finally (i) and (vii) show that the $f_{n}$ converge uniformly to a function $f^{*} \in L(U)$ satisfying $\left\|f^{*}-f\right\|<\epsilon$, and $f^{*}=g^{*}+b^{*} \in H+L_{Q}$. Hence $f$ is in the uniform closure of $H+L_{Q}$, and the proof is complete.

11.5 Corollary. Let $H$ and $Q$ be as in Theorem 11.4. If $E$ is any subset of $Q$, then $H+L_{E}$ is a closed subalgebra of $L(U)$.

Proof. That $H+L_{E}$ is closed follows from 6.2 and 10.1. To see that $H+L_{E}$ is an algebra, it suffices to show that $b H \subseteq H+L_{E}$ for every $b \in L_{V}$, where $V$ is an open neighborhood of $E$ in $\partial U$. However, in this case $V \cap Q$ is $\sigma$-compact, so that, by 7.4, $V \cap Q$ satisfies the hypotheses of 11.4 . This yields $b H \subseteq H+L_{V \cap Q} \subseteq H+L_{E}$.

Theorems 9.2 and 9.8 show that the conclusions of 11.4 and 11.5 hold for the algebras $H^{\infty}(\sigma)$ and $H^{\infty}(\mu)$. We state this result explicitly, for emphasis. 
11.6 Theorem. Let $\mu$ be barmonic measure on $\partial U$, and let $H^{\infty}(\mu)$ be the weak-star closure of $A(U)$ in $L^{\infty}(\mu)$. If $E$ is an arbitrary subset of $\partial U$, then $H^{\infty}(\mu)+L_{E}^{\infty}(\mu)$ is a closed subalgebra of $L^{\infty}(\mu)$. If moreover $E$ is $\sigma$-compact, then $H^{\infty}(\mu)+L_{E}^{\infty}(\mu)$ consists of precisely the functions in $L^{\infty}(\mu)$ which belong to $H^{\infty}(\mu)$ on each fiber of the maximal ideal space of $L^{\infty}(\mu)$ over points of $E$.

12. The algebra $H^{\infty}(d \theta)+L_{E}^{\infty}(d \theta)$. We now consider the case in which $U=\Delta$ is the open unit disc in the complex plane. The harmonic measure on $\partial \Delta$ is the normalized angular measure $d \theta$. Theorem 11.6 asserts that $H^{\infty}(d \theta)+$ $L_{E}^{\infty}(d \theta)$ is a closed subalgebra of $L^{\infty}(d \theta)$, for any subset $E$ of $\partial \Delta$.

In [6] Douglas considers subalgebras $B$ of $L^{\infty}(d \theta)$ with the following property: there is a family $\mathcal{F}$ of inner functions in $H^{\infty}(d \theta)$ such that $B$ is the algebra generated by $H^{\infty}(d \theta)$ and the complex conjugates of the functions in $\mathcal{F}$. Douglas proves that if $B$ is any such algebra, if $\mathcal{B}$ is the operator algebra on $H^{2}(d \theta)$ generated by the Toeplitz operators $T_{f}, f \in B$, and if $\mathcal{C}$ is the commutator ideal of $B$, then the correspondence $f \rightarrow T_{f}+\mathcal{C}$ is an isometric algebra isomorphism of $B$ onto $\mathfrak{B} / \mathcal{C}$.

It is easy to see that Sarason's algebra $H^{\infty}(d \theta)+C(\partial \Delta)$ is such an algebra, since it is generated by $H^{\infty}(d \theta)$ and the complex conjugate of the coordinate function $Z$. In this case, the commutator ideal $\mathcal{C}$ is precisely the ideal of compact operators on $H^{2}(d \theta)$.

A result of Douglas and Rudin [7] asserts that $L^{\infty}(d \theta)$ is generated by $H^{\infty}(d \theta)$ and the complex conjugates of the inner functions in $H^{\infty}(d \theta)$. We intend to prove the analogous theorem for the algebras $H^{\infty}(d \theta)+L_{E}^{\infty}(d \theta)$. This is easy to do, once we prove the following extension of the main result of [7].

12.1 Theorem. Let $E$ be an arbitrary subset of the unit circle $\partial \Delta$. Then every unimodular function in $L_{E}^{\infty}(d \theta)$ can be approximated uniformly by functions of the form $F / G$, where $F$ and $G$ are Blaschke products which are analytic on $E$.

Before beginning the proof, we mention that the case $E=\partial \Delta$ is elementary, and has appeared in the literature (cf. [16]). In this case, the theorem asserts that every unimodular function $u \in C(\partial \Delta)$ can be approximated uniformly by quotients of finite Blaschke products. To see this, express $u$ in the form $z^{n} v^{2}$ for some $v \in C(\partial \Delta)$, approximate $v$ uniformly by a rational function $b$, and observe that $z^{n} b(z) / \overrightarrow{b(1 / \bar{z})}$ is a quotient of finite Blaschke products and is close to $u$.

Since every unimodular function in $L_{E}^{\infty}(d \theta)$ can be approximated uniformly by unimodular functions which are continuous on an open set containing $E$, we may as well assume that $E$ is an open subset of $\partial \Delta$. It will be convenient to work with the upper half plane instead of the unit disc, so that we will assume 
henceforth that $E$ is an open subset of the extended real line, and that $\infty \in E$.

The proof given by Douglas and Rudin shows that every unimodular function in $L_{E}^{\infty}$ which is constant on $E$ can be approximated uniformly by a quotient of Blaschke products which are analytic on $E$. It suffices then to show that every continuous unimodular function on $E$ can be approximated uniformly on $E$ by a quotient of Blascbke products which are analytic on $E$. The next lemma shows that we need approximate only functions with a bounded argument.

12.2 Lemma. If $f$ is a continuous unimodular function on $E$, there are Blaschke products $B_{1}$ and $B_{2}$ which are analytic on $E$, such that $\arg \left(f B_{1} / B_{2}\right)$ bas a bounded continuous brancb on $E$.

Proof. We can find two step functions $\chi_{1}$ and $\chi_{2}$ defined on $E$, which are nondecreasing on each interval in $E$, and which take only values which are integral multiples of $2 \pi$, so that $\chi_{1}-\chi_{2}+\arg f$ is bounded. Let $\left\{x_{n}\right\}$ be the points where $\chi_{1}$ jumps, and let $\left\{y_{n}\right\}$ be the points where $\chi_{2}$ jumps. Let $a_{n}=x_{n}+i \alpha_{n}$ and $b_{n}=y_{n}+i \beta_{n}$, where $\alpha_{n}, \beta_{n}>0$ are small numbers to be fixed. We observe that as $\alpha_{n} \rightarrow 0$, arg $\left(\left(x-a_{n}\right) /\left(x-\bar{a}_{n}\right)\right)$ converges to a step function whose only discontinuity is a jump of $2 \pi$ at $x_{n}$. The same goes for $\beta_{n}$. So if we choose $a_{n}$ and $\beta_{n}$ small enough, the Blaschke products $B_{1}(z)=$ $\Pi\left(\left(z-a_{n}\right) /\left(z-\overline{a_{n}}\right)\right)$ and $B_{2}(z)=\Pi\left(\left(z-b_{n}\right) /\left(z-\bar{b}_{n}\right)\right)$ will converge, and $\arg \left(f B_{1} / B_{2}\right)$ will have a bounded continuous branch on $E$. We can assume that the $x_{n}$ and $y_{n}$ do not cluster in $E$, so that $B_{1}$ and $B_{2}$ will be analytic on $E$. That does it.

The approximation of continuous unimodular functions on $E$ with bounded argument is accomplished in the next two lemmas.

12.3 Lemma. Let $0<\epsilon, \delta<1$, and let $g$ be a continuous real-valued function on $\mathbf{R}$ which is zero outside a closed interval $I$ of length $|I|$. Let $V$ be a neigbborbood of $I$ in $R$. Then we can find finite Blascbke products $B_{1}, B_{2}$ sucb that

(1) $\left|g-\arg \left(B_{1} / B_{2}\right)\right|<4 \epsilon(1+\|g\|)$ on $\mathbf{R}$.

(2) $\left|\arg \left(B_{1} / B_{2}\right)\right|<\delta$ outside $V$.

(3) The sum of the imaginary parts of the zeros of $B_{1}$ and $B_{2}$ is less than $A(\epsilon)|I|\|g\|$, where $A(\epsilon)$ depends only on $\epsilon$.

Moreover the zeros of $B_{1}$ and $B_{2}$ can be chosen to lie in any prescribed neigbborbood of I in C.

Proof. Consider the function

$$
\psi(x)=2 \int_{x-\epsilon^{2}}^{x+\epsilon^{2}} \frac{d t}{1+t^{2}}, \quad x \in \mathbf{R} .
$$


Evidently $\psi$ is an even function, $\psi>0, \psi(x)$ increases for $x<0, \psi(x)$ decreases for $x>0$, and $\psi(0)<4 \epsilon^{2}$. Since

$$
\begin{aligned}
\int_{x-\epsilon^{2}}^{x+\epsilon^{2}} \frac{d t}{1+t^{2}} & =\arg \left(x-\epsilon^{2}+i\right)-\arg \left(x+\epsilon^{2}+i\right) \\
& =\arg \left(x+\epsilon^{2}-i\right)-\arg \left(x-\epsilon^{2}-i\right),
\end{aligned}
$$

we can express $\psi$ as the argument of the quotient of two simple Blaschke products with zeros at $x-\epsilon^{2}+i$ and $x+\epsilon^{2}+i$ :

$$
\psi(x)=\arg \frac{\left(x-\epsilon^{2}+i\right)}{\left(x-\epsilon^{2}-i\right)} \frac{\left(x+\epsilon^{2}-i\right)}{\left(x+\epsilon^{2}+i\right)} .
$$

One computes easily that $\|\psi\|_{1}=4 \pi \epsilon^{2}$, so that the functions $\psi_{\rho}(x)=$ $\left(1 / 4 \pi \epsilon^{2} \rho\right) \psi(x / \rho), x \in \mathbf{R}, \rho>0$, form an approximate identity as $\rho \rightarrow 0$.

We can assume that $g \geq 0$. Let $\eta=4 \pi \epsilon \rho$, and choose $\rho$ so small that

$$
\begin{aligned}
& \left\|\psi_{\rho} * g-g\right\|_{\infty}<\epsilon, \\
& \quad \mid g(s)-g(t)<\epsilon \text { if }|s-t|<\eta .
\end{aligned}
$$

Partition $\mathbf{R}$ into contiguous intervals $I_{j}$ of length $\eta$ and left endpoint $a_{j}$. Write

$$
h(x)=\sum_{j=-\infty}^{\infty}\left[\frac{g\left(a_{j}\right)}{\epsilon}\right] 4 \pi \epsilon^{2} \rho \psi_{\rho}\left(x-a_{j}\right),
$$

where [.] denotes the greatest integer function. Then $b(x)=\arg B_{1}(x) / B_{2}(x)$, where $B_{1}$ and $B_{2}$ are Blaschke products with zeros at $a_{j}-\epsilon^{2} \rho+i \rho$, and $a_{j}+\epsilon^{2} \rho+i \rho$ respectively, with multiplicity $\left[g\left(a_{j}\right) / \epsilon\right]$. So the sum of the imaginary parts of these zeros does not exceed $\Sigma\left(g\left(a_{j}\right) / \epsilon\right) \rho=\left(1 / 4 \pi \epsilon^{2}\right) \Sigma \eta g\left(a_{j}\right)$. Since there are at most $|I| / \eta$ points $a_{j}$ at which $g$ does not vanish, this sum is bounded by $|I|\|g\| / 4 \pi \epsilon^{2}$, and (3) is valid.

Let $d$ be the distance from $\partial V$ to $I$. If $x \in \mathbf{R} \backslash V$, then $|b(x)| \leq$ $\Sigma g\left(a_{j}\right) \eta \psi_{\rho}\left(x-a_{j}\right) \leq \Sigma \eta g\left(a_{j}\right) \psi_{\rho}(d) \leq|I|\|g\| \psi_{\rho}(d)$. Since $\psi_{\rho}(d) \rightarrow 0$ as $\rho \rightarrow 0$, we can obtain (2) by taking $\rho$ sufficiently small.

To verify (1), it will suffice to show that

$$
\left\|b-\psi_{\rho} * g\right\| \leq \epsilon^{2}+2 \epsilon+\epsilon\|g\| \text {. }
$$

For this, estimate

where

$$
\left|b(x)-\left(\psi_{\rho} * g\right)(x)\right| \leq T_{1}+T_{2}+T_{3}
$$




$$
\begin{aligned}
& T_{1}=\sum\left|\left[\frac{g\left(a_{j}\right)}{\epsilon}\right]-\frac{g\left(a_{j}\right)}{\epsilon}\right| 4 \pi \epsilon^{2} \rho \psi_{\rho}\left(x-a_{j}\right), \\
& T_{2}=\sum g\left(a_{j}\right)\left|\eta \psi_{\rho}\left(x-a_{j}\right)-\int_{I_{j}} \psi_{\rho}(x-y) d y\right|, \\
& T_{3}=\sum \int_{I_{j}} \psi_{\rho}(x-y)\left|g\left(a_{j}\right)-g(y)\right| d y .
\end{aligned}
$$

Here $T_{3}$ is easy to estimate

$$
T_{3} \leq \int_{-\infty}^{\infty} \psi_{\rho}(x-y) \epsilon d y=\epsilon .
$$

Suppose $x$ lies in the interval $I_{k}$. Since $\psi_{\rho}(t)$ is even and decreasing for $t>0$,

$$
\begin{aligned}
& \sum_{j}\left|\eta \psi_{\rho}\left(x-a_{j}\right)-\int_{I_{j}} \psi_{\rho}(x-y) d y\right| \\
& \leq \sum_{j<k}\left[\eta \psi_{\rho}\left(x-a_{j+1}\right)-\eta \psi_{\rho}\left(x-a_{j}\right)\right] \\
& \quad+\sum_{j>k}\left[\eta \psi_{\rho}\left(x-a_{j}\right)-\eta \psi_{\rho}\left(x-a_{j+1}\right)\right]+\left|\eta \psi_{\rho}\left(x-a_{k}\right)-\int_{I_{k}} \psi_{\rho}(x-y) d y\right| \\
& \leq 3 \eta \psi_{\rho}(0) \leq \eta / 4 \pi \rho=\epsilon .
\end{aligned}
$$

Hence $T_{2} \leq \epsilon\|g\|$, and

$$
T_{1} \leq \epsilon \sum \eta \psi_{\rho}\left(x-a_{j}\right) \leq \epsilon\left(\epsilon+\int_{-\infty}^{\infty} \psi_{\rho}|t| d t\right)=\epsilon^{2}+\epsilon .
$$

That establishes $(1)^{\prime}$, and the proof is complete.

12.4 Lemma. If $g$ is a bounded continuous real-valued function on $E$, and if $\epsilon>0$, there are Blaschke products $B_{1}$ and $B_{2}$, analytic across $E$, such that $\left|g-\arg \left(B_{1} / B_{2}\right)\right|<\epsilon$.

Proof. We can assume that $g$ vanishes near $\infty$. Put $E_{0}=\{x \in E: d(x, \mathbf{R} \backslash E)$ $<1 / 2\}, E_{n}=\left\{x \in E: 2^{-n-1}<d(x, \mathbf{R} \backslash E)<2^{-n+1}\right\}, n \geq 1$. We can write $g=\sum_{n=0}^{\infty} g_{n}$, where $g_{n}$ is real-valued and continuous on $R, g_{n}$ vanishes outside a compact subset of $E_{n}$, and $\left\|g_{n}\right\| \leq\|g\|$.

Each $E_{n}$ is a finite union of open intervals. By applying Lemma 12.3 to each component of $E_{n}$, we can find finite Blaschke products $B_{1}^{(n)}$ and $B_{2}^{(n)}$ such that

$$
\begin{aligned}
\mid g_{n}-\arg \left(B_{1}^{(n)} / B_{2}^{(n)}\right) & <\epsilon / 4 \text { on } \mathbf{R}, \\
& \left|\arg \left(B_{1}^{(n)} / B_{2}^{(n)}\right)\right|<\epsilon / 2^{n+2} \text { on } \mathbf{R} \backslash E_{n^{\prime}}
\end{aligned}
$$


and such that the sum of the imaginary parts of the zeros of $B_{1}^{(n)}$ and $B_{2}^{(n)}$ does not exceed $A(\epsilon)\left|E_{n}\right|\|g\|$, where $\left|E_{n}\right|$ is the length of $E_{n}$. We can moreover assume that the zeros of $B_{1}^{(n)}$ and $B_{2}^{(n)}$ lie in the $2^{-n}$-neighborhood of $E_{n}$. Since $\Sigma\left|E_{n}\right|<\infty$, the conditions on the zeros of $B_{1}^{(n)}$ and $B_{2}^{(n)}$ show that $B_{1}=\Pi B_{1}^{(n)}$ and $B_{2}=\Pi B_{2}^{(n)}$ are convergent Blaschke products which are analytic on $E$. Since no point $x \in E$ is contained in more than two of the $E_{j}$ 's, we have

$$
\sum_{n=0}^{\infty}\left|g_{n}(x)-\arg \left(B_{1}^{(n)}(x) / B_{2}^{(n)}(x)\right)\right|<2(\epsilon / 4)+\sum_{n=0}^{\infty} \epsilon / 2^{n+2}=\epsilon .
$$

Hence $\left|g(x)-\arg \left(B_{1}(x) / B_{2}(x)\right)\right|<\epsilon$ for all $x \in E$. That proves the lemma, and the proof of Theorem 12.1 is now complete.

12.5 Corollary. Let $E$ be an arbitrary subset of $\partial \Delta$. Then every function in $H^{\infty}(d \theta)+L_{E}^{\infty}(d \theta)$ can be approximated uniformly by functions of the form $\vec{F} g$, where $g \in H^{\infty}(d \theta)$ and $F$ is a Blaschke product which is analytic on $E$.

Proof. The functions $\vec{F} g$, where $F$ and $g$ are as above, form a subalgebra of $H^{\infty}(d \theta)+L_{E}^{\infty}(d \theta)$. The uniform closure of this algebra includes $H^{\infty}(d \theta)$ and the unimodular functions in $L_{E}^{\infty}(d \theta)$, and hence all of $H^{\infty}(d \theta)+L_{E}^{\infty}(d \theta)$.

13. Some open problems. Many of the results concerning $H^{\infty}(U)$ whose proofs depend on Vitushkin's constructive techniques (cf. especially [12]) are valid also for stable subalgebras of $H^{\infty}(U)$. However, it is not known whether the analogue of the cluster value lemma for $H^{\infty}(U)$ is valid for stable algebras. Using the same notation as in Lemma 3.2, we ask the following:

13.1 Problem. For which closed stable subalgebras $H$ of $H^{\infty}(U)$ is it true that, for all $f \in H, f\left(\pi_{\lambda}(H)\right)$ coincides with the cluster set of $f$ at $\lambda$ (from $U$ )? In particular, is this true for the algebra $H^{\infty}(\sigma)$ introduced in $\$ 9$ ?

The proof of Theorem 1.1, characterizing pointwise bounded density in terms of a distance estimate, depends on the fact that the distances are measured in the supremum norm over all of $U$ (cf. the proof of Theorem 2.2). If $b \in C(\partial U$ ), then it is easy to define $d(b, A(U))$ and $d\left(b, H^{\infty}(U)\right)$.

13.2 Problem. Is $A(U)$ pointwise boundedly dense in $H^{\infty}(U)$ if and only if $d(b, A(U))=d\left(b, H^{\infty}(U)\right)$, all $b \in C(\partial U)$ ?

It is easy to construct $U$, and a closed set $E \subset \partial U$, such that there is no constant $c$ satisfying

$$
d\left(b, H_{E}^{\infty}(U)\right) \leq c d\left(b, H^{\infty}(U)\right), \text { all } b \in L_{E} .
$$

This amounts to the existence of a measure in $\left(H_{E}^{\infty}(U)\right)^{\perp}$ which cannot be "lifted" to a measure in $\left(H^{\infty}(U)\right)^{\perp}$. The question is whether the existence of a constant 
c satisfying $(*)$ already implies that we can take $c=1$. In particular, we ask the following.

13.3 Problem. Is $A(U)$ pointwise boundedly dense in $H^{\infty}(U)$ if and only if $H^{\infty}(U)+C(\bar{U})$ is a closed subspace of $L(U)$ ?

The iteration procedures we have used have restricted the validity of some of the main results to $\sigma$-compact sets, and it is natural to ask whether these results can be extended to arbitrary sets.

13.4 Problem. Is there a tractable characterization of all subsets $E$ of $\partial U$ such that $d\left(b, H_{E}\right)=d(b, H)$ for all $b \in L_{E}$ ? If $\cdot H+L_{E}$ is a closed subalgebra of $L(U)$, does it necessarily consist of all $f \in L(U)$ such that $\left.\left.f\right|_{\pi_{\lambda}(H)} \in H\right|_{\pi_{\lambda}(H)}$ for all $\lambda \in E$ ?

This latter question seems difficult, even for the algebra $H^{\infty}(\Delta)$.

Finally, related to $\$ \$ 7$ and 8 , we have the following question:

13.5 Problem. Is it true that $\widetilde{H}_{E}$ always coincides with $H_{E}$ ?

This is probably not true. But if one could prove this for the algebra $H^{\infty}(U)$, one could deduce strong results on the semi-additivity of analytic capacity (cf. $\$ 8$ of [3]).

\section{BIBLIOGRAPHY}

1. V. M. Adamjan, D. Z. Arov and M. G. KreǏn, Infinite Hankel matrices and generalized problems of Carathéodory-Fejer and F. Riesz, Funkcional. Anal. i Priložen, 2 (1968), no. 1, 1-19. (Russian) MR 38 \#2591.

2. A. M. Davie, Bounded approximation and Dirichlet sets, J. Functional Analysis 6 (1970), 460-467. MR 43 \#925.

3. - Analytic capacity and approximation problems, Trans. Amer. Math. Soc. 171 (1972), 409-444.

4. - Bounded limits of analytic functions, Proc. Amer. Math. Soc. 32 (1972), $127-133$.

5. J. Detraz, Étude du spectre d'algèbres de fonctions analytiques sur le disque unité, C. R. Acad. Sci. Paris Sér. A-B 269 (1969), A833-A835. MR 40 \#7812.

6. R. Douglas, On the spectrum of Toeplitz and Wiener-Hopf operators, Proc. Conf. Abstract Spaces and Approximation (Oberwolfach, 1968), Birkhäuser, Basel, 1969, pp. 53-66. MR $41 \# 4274$.

7. R. Douglas and W. Rudin. Approximation by inner functions, Pacific J. Math. 31 (1969), 313-320.

8. S. Fisher, Bounded approximation by rational functions, Pacific J. Math. 28 (1969), 319-326. MR $39 \# 1663$.

9. T. Gamelin, Norm compactness of representing measures for $R(K)$, J. Functional Analysis 3 (1969), 495-500. MR 39 \#782.

10. - Uniform algebras, Prentice-Hall, Englewood Cliffs, N. J., 1969.

11. - Localization of the corona problem, Pacific J. Math. 34 (1970), 73-81. MR $43 \# 2482$.

12. T. Gamelin and J. Garnett, Distinguished homomorphisms and fiber algebras, Amer. J. Math. 42 (1970), 455-474.

13. - - Pointwise bounded approximation and Dirichlet algebras, J. Functional Analysis 8 (1971), 360-404.

14. - Uniform approximation to bounded analytic functions, Rev. Un. Mat. Argentina (to appear). 
15. - Bounded approximation by rational functions, Pacific J. Math (to appear).

16. H. Helson and D. Sarason, Past and future, Math. Scand. 21 (1967), 5-16. MR 38 \#5282.

17. D. Sarason, Generalized interpolation in $H^{\infty}$, Trans. Amer. Math. Soc. 127 (1967), 179-203. MR 34 \#8193.

18. A. Stray, An approximation theorem for subalgebras of $H^{\infty}$, Pacific J. Math. 35 (1970), 511-515. MR 43 \#2515.

19. A. G. Vituškin, Analytic capacity of sets in problems of approximation theory, Uspehi Mat. Nauk 22 (1967), no. 6 (138), 141-199= Russian Math. Surveys 22 (1967), no. 6, 139-200. MR 37 \#5404.

20. L. Zalcman, Bounded analytic functions on domains of infinite connectivity, Trans. Amer. Math. Soc. 144 (1969), 241-269. MR 40 \#5884.

DEPARTMENT OF MATHEMATICS, UNIVERSITY OF CALIFORNIA, LOS ANGLELES, CALIFORNIA 90024

Current address (A. M. Davie): Mathematical Institute, 20 Chambers Street, Edinburgh, EH1 $1 \mathrm{HZ}$, Scotland 